\title{
High and Low Levels of an NTRK2-Driven Genetic Profile Affect Motor- and Cognition-Associated Frontal Gray Matter in Prodromal Huntington's Disease
}

\author{
Jennifer A. Ciarochi ${ }^{1}$ (i), Jingyu Liu ${ }^{2}$, Vince Calhoun ${ }^{2,3}$, Hans Johnson ${ }^{4}$ (i), Maria Misiura ${ }^{5}$, \\ H. Jeremy Bockholt ${ }^{2}$, Flor A. Espinoza ${ }^{2}$, Arvind Caprihan ${ }^{2}$, Sergey Plis ${ }^{2}$, Jessica A. Turner ${ }^{1,5, *}$, \\ Jane S. Paulsen $4,6,7$ (iD and the PREDICT-HD Investigators and Coordinators of the Huntington \\ Study Group ${ }^{\dagger}$ \\ 1 Neuroscience Institute, Georgia State University, Atlanta, GA 30302, USA; jciarochi1@student.gsu.edu \\ 2 The Mind Research Network, Albuquerque, NM 87106, USA; jliu@mrn.org (J.L.); vcalhoun@mrn.org (V.C.); \\ jbockholt@mrn.org (H.J.B.); fespinoza@mrn.org (F.A.E.); acaprihan@mrn.org (A.C.); splis@mrn.org (S.P.) \\ 3 Department of Electrical and Computer Engineering, University of New Mexico, Albuquerque, \\ NM 87131, USA \\ 4 Iowa Mental Health Clinical Research Center, Department of Psychiatry, University of Iowa, Iowa City, \\ IA 52242, USA; hans-johnson@uiowa.edu (H.J.); jane-paulsen@uiowa.edu (J.S.P.) \\ 5 Department of Psychology, Georgia State University, Atlanta, GA 30302, USA; mmisiura1@student.gsu.edu \\ 6 Department of Neurology, University of Iowa, Iowa City, IA 52242, USA \\ 7 Department of Psychology, University of Iowa, Iowa City, IA 52242, USA \\ * Correspondence: jturner63@gsu.edu; Tel.: +1-404-413-6211 \\ + Detailed information in Author Contribution part.
}

Received: 16 May 2018; Accepted: 20 June 2018; Published: 22 June 2018

\begin{abstract}
This study assessed how BDNF (brain-derived neurotrophic factor) and other genes involved in its signaling influence brain structure and clinical functioning in pre-diagnosis Huntington's disease (HD). Parallel independent component analysis (pICA), a multivariate method for identifying correlated patterns in multimodal datasets, was applied to gray matter concentration (GMC) and genomic data from a sizeable PREDICT-HD prodromal cohort $(N=715)$. pICA identified a genetic component highlighting NTRK2, which encodes BDNF's TrkB receptor, that correlated with a GMC component including supplementary motor, precentral/premotor cortex, and other frontal areas $(p<0.001)$; this association appeared to be driven by participants with high or low levels of the genetic profile. The frontal GMC profile correlated with cognitive and motor variables (Trail Making Test A $(p=0.03)$; Stroop Color $(p=0.017)$; Stroop Interference $(p=0.04)$; Symbol Digit Modalities Test $(p=0.031)$; Total Motor Score $(p=0.01))$. A top-weighted NTRK2 variant (rs2277193) was protectively associated with Trail Making Test B $(p=0.007)$; greater minor allele numbers were linked to a better performance. These results support the idea of a protective role of NTRK2 in prodromal HD, particularly in individuals with certain genotypes, and suggest that this gene may influence the preservation of frontal gray matter that is important for clinical functioning.
\end{abstract}

Keywords: Huntington's disease; brain-derived neurotrophic factor; tropomyosin receptor kinase B; supplementary motor; independent component analysis 


\section{Introduction}

\subsection{Huntington's Disease}

Huntington's disease (HD) is a progressive, heritable condition characterized by chorea (involuntary motion) as well as cognitive alterations spanning executive functioning, working memory, olfactory and facial recognition, and emotional processing [1]. HD, along with Alzheimer's disease (AD) and Parkinson's disease (PD), is a proteinopathy distinguished by regionally-selective neuronal death and protein misfolding that manifests as expanded huntingtin in HD, Lewy bodies in PD, and $\beta$-amyloid plaques in AD [2]. Unfortunately, across these conditions limited treatment options and no known cures are available. However, their shared features have sparked speculation about common underlying mechanisms, and the delayed-onset of these disorders raises the appealing possibility of developing treatments that postpone onset indefinitely, effectively eradicating the disease.

A promising way to identify treatment targets is to characterize the earliest changes before the onset of HD. Motor impairments associated with HD, such as dystonia and chorea, often lead to diagnosis because their disruptiveness prompts affected individuals to seek medical attention. However, cognitive symptoms and alterations in brain volume and morphology are already present more than a decade before diagnosis, during a period known as the prodrome [3]. In keeping with this, PREDICT-HD is a multi-site research study aiming to identify the earliest changes in the HD prodrome, with the hopes of identifying targets for the earliest possible interventions [3]. PREDICT-HD has amassed a comprehensive dataset of genomic, structural and functional magnetic resonance imaging (MRI and fMRI), diffusion tensor imaging (DTI), cognitive and motor assays, cytosine-adenine-guanine (CAG)-repeat information, and demographic variables from over 1449 prodromal HD and control participants, including longitudinal data from over 900 participants.

Although there is no cure for HD, its cause is known. An abnormally large cytosine-adenine-guanine (CAG) expansion ( $\geq 36$ repeats) at an HTT exon 1 locus determines future HD development. The HTT gene encodes huntingtin protein, which is widely expressed in the brain and central nervous system [4]. Abnormally-expanded HTT encodes mutant huntingtin (mHTT), which compromises numerous cellular processes including endocytosis and secretion, calcium homeostasis [5], glutamatergic synaptic functioning [6], vesicular transport [7], mitochondrial functioning [8], p53 signaling [7], apoptosis, and transcription [9].

\subsection{Effects of Multiple Genes and Variants}

Both within and outside the realm of huntingtin's interactions, several lines of evidence implicate non-HTT factors as modulators of prodromal progression and HD onset. Moreover, the reduced genetic complexity of HD makes it tractable to disentangle onset-protection and susceptibility factors. HTT CAG-expansion length considerably influences age at diagnosis and can be used to estimate the age of, or time to, HD onset. Despite strong prediction accuracy for many prodromal individuals, some outcomes deviate from expectations. For example, one PREDICT-HD participant with 44 CAG-repeats lacked positive diagnosis at the age of 71 years, and 13 participants with $<41$ repeats reached the age of 70 years with no diagnosis. HD onset prediction (based on age and CAG-repeat number) is most accurate in individuals with $>44$ repeats and increasingly variable as the repeat number decreases, and different disease progression rates are often observed in persons with the same number of CAG-repeats. These examples highlight the onset variability and suggest that additional genetic factors may promote or suppress HD conversion (especially at lower CAG-repeat numbers), yet little is known about non-HTT genetic factors that account for variability in the rapidity and severity of HD symptoms and onset.

The influence of such factors is likely also reflected by differences in brain structure and clinical functioning throughout the prodrome. Known polygenetic neural effects suggest that multiple genes may modulate decline; this is in keeping with the prevailing common disease-common variant model, which posits that the combined effects of multiple common nucleotide variants, or single 
nucleotide polymorphisms (SNPs), with weak individual effects may confer disease susceptibility or resistance [10]. At an individual level, these polymorphisms may occur in several, sometimes interacting genes, bestowing weak enough effects to fall below statistical thresholds and avoid elimination via natural selection [10]. We observe similar covariance in the brain; even in disorders with regionally concentrated damage, multiple brain regions and cell types are usually affected.

\subsection{Benefits of Multivariate Methods}

Interactions among multiple genes can have complex effects on disease phenotypes [10]. Univariate methods such as genome-wide association studies (GWAS) have dominated large-scale human genetic studies, despite an inability to capture this important covariation $[10,11]$. Univariate tests require tens of thousands of participants, which can be impossible to achieve in rare clinical populations, and must correct for many statistical tests. These stringent statistical standards can result in an overshadowing of small-to-moderate genetic effects and obscured interpretation of impacted biological pathways, as results generally consist of a few of the most significant genes, each of which is involved in multiple cellular processes and pathways. For these reasons, multivariate methods may be more suitable for extensive genetic studies, especially in rare clinical populations with fewer available study participants. Rather than assessing related points, multivariate tests find interrelated patterns and can detect weak effects in high-dimensional data.

\subsection{Parallel Independent Component Analysis (pICA)}

Combined effects of nucleotide-level differences (or SNPs) on gray matter concentration (GMC) across the brain can be assessed across the genome and in candidate genes using the multivariate method of parallel ICA (pICA) $[10,11]$. Through the simultaneous analysis of multimodal data, pICA can isolate groups of correlated SNPs into novel, maximally-independent networks that affect patterns of GMC in a population. In other words, a person whose genome aligns with a pICA SNP profile that is correlated with a GMC profile will also likely display a brain structure consistent with that GMC profile. pICA has been successfully applied to other clinical populations, including schizophrenia and Alzheimer's disease [12,13], which share key features with HD such as delayed onset, regional and cellular selectivity of atrophy, and cognitive abnormalities.

Like other multivariate methods, pICA is optimal for examining multi-gene interactions because it considers the cumulative effects of changes at multiple loci, likely accounting for more variation than the strongest changes in single genes [10]. Consequently, it requires fewer statistical tests than univariate Genome-Wide Scanning (GWS) analyses ( $<30$ compared to hundreds of thousands). An additional advantage of pICA is that it permits both hypothesis-driven testing (via inclusion of reference SNPs) and the exploration of new and unexpected patterns (disease-related or otherwise) that connect genes and their expression to brain structure and function. These advantages allow affected pathways and gene networks to be more thoroughly defined.

\subsection{Brain-Derived Neurotrophic Factor (BDNF)-Signaling Genes (a Candidate Pathway)}

Genes that interact with mHTT and are involved in mHTT-compromised processes are strong candidate HD-progression mediators. One such target is brain-derived neurotrophic factor (BDNF). BDNF mediates neurogenesis, and accumulating evidence suggests its importance in HD development and onset $[9,14]$. BDNF co-localizes with huntingtin in 99\% of pyramidal motor cortical neurons and $75 \%$ of striatal neurons, and is necessary for healthy cortico-striatal synaptic activity and striatal GABA-ergic medium spiny neuron (MSN) survival [9].

mHTT interferes with BDNF transcription and vesicular transport (Figure 1). (a) Huntingtin enhances BDNF microtubule transport by binding to HAP1, which engages vesicle transport proteins. mHTT binds too tightly to HAP1, inhibiting transport. (b) Huntingtin stimulates transcription from the BDNF exon II promoter, which is $60 \%$ less active in cells overexpressing mHTT [9]. Like many neuronal-gene promoters, BDNF is regulated by repressor element $1 /$ neuron-restrictive silencer 
element (RE1/NRSE), which is modulated by RE1 silencing transcription factor/neuron-restrictive silencer factor (REST/NRSF) [7]. REST is a neuron-specific, master gene repressor that binds to BDNF promoter II RE1 sites and recruits a co-repressor complex that includes Sin3A and REST co-repressor (coREST). Huntingtin indirectly sequesters REST in the cytoplasm by interacting with HAP1 and REST-interacting LIM domain protein (RILP), which directly binds REST/NRSF to mediate translocation to the nucleus. mHTT disrupts this complex by failing to isolate REST in the cytoplasm, leading to its increase in the nucleus and consequent recruitment of transcriptional repressors.
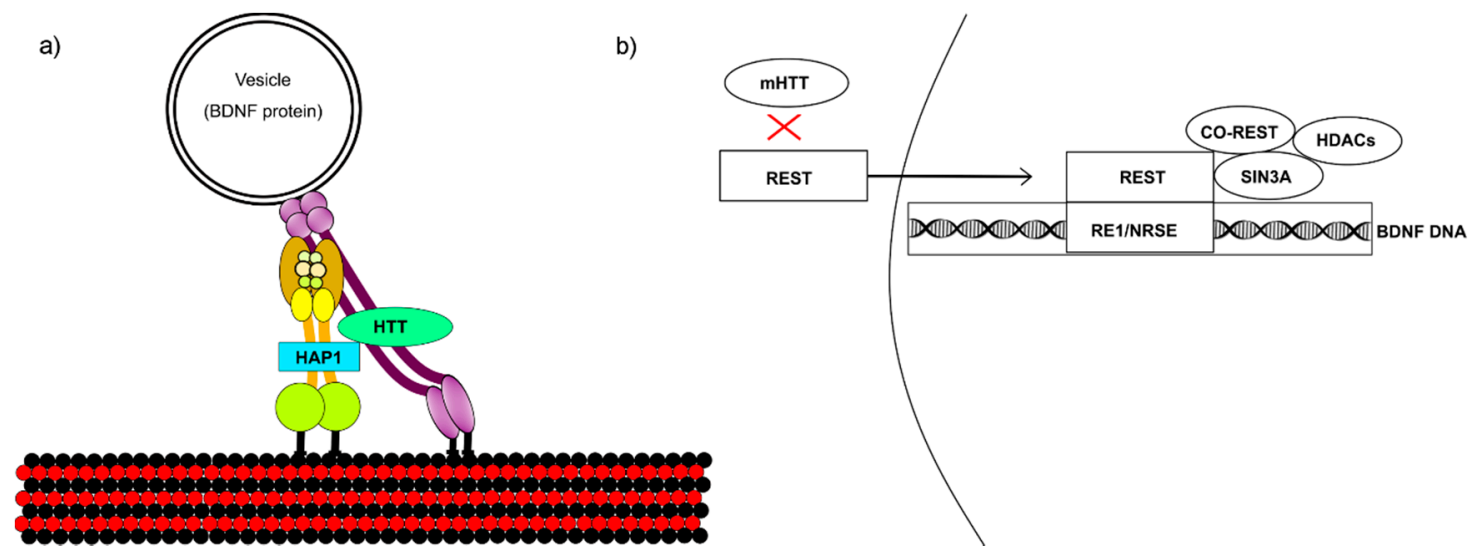

Figure 1. Mutant huntingtin (mHTT) affects (a) brain-derived neurotrophic factor (BDNF) vesicular transport across microtubules and (b) BDNF transcription. (a) Huntingtin binds to HAP1 to recruit vesicular transport proteins. The dynein complex is shown in yellow and the dynactin complex in purple. Together, these proteins enable retrograde vesicular transport, which is disrupted by mHTT binding too tightly to HAP1. (b) Huntingtin sequesters RE1 silencing transcription factor (REST) in the cytoplasm, inhibiting its accumulation in the nucleus. If mHTT fails to sequester REST, this leads to its nuclear buildup and consequently reduces BDNF transcription.

BDNF is particularly relevant to prodromal HD. Compared to controls, asymptomatic HD transgenic mice have reduced striatal BDNF that is lower at higher CAG-repeat numbers, indicating a prodromal BDNF deficit [14]. Further BDNF reduction in these mice lowers onset age and worsens motor symptoms, correlating with brain morphology changes. Furthermore, BDNF deficiency only modestly contributes to early-life MSN survival, yet significantly reduces MSNs (by 35\%) in later life, consistent with delayed HD onset. Interestingly, the striatum does not produce its own BDNF, but receives $\sim 95 \%$ from the cortex and the remainder from the substantia nigra pars compacta, amygdala, and thalamus [14]. Thus, prodromal BDNF suggests possible early deficits in regions that supply striatal BDNF. Given that BDNF mRNA and protein reductions are present in prodromal and diagnosed $\mathrm{HD}$ and are causally related to symptom severity and HD onset, factors that interact with BDNF likely confer additional symptom and onset variability.

\subsection{The Present Study}

Using parallel ICA with reference (pICAr), this study leveraged legacy imaging, genomic, and clinical data from PREDICT-HD to investigate how SNPs in BDNF-signaling genes impact clinical functioning and patterns of brain morphology in prodromal HD. As an expansion of the pICA results, we assessed the effects of four individual SNPs in NTRK2 (which encodes BDNF's TrkB receptor) on frontal gray matter and clinical functioning. Profiles including SNPs from multiple genes were anticipated to correlate with gray matter concentration profiles and clinical functioning with maximum effects in BDNF-related, HD-compromised pathways. 


\section{Materials and Methods}

\subsection{Participants}

A cohort of 715 expansion-positive ( $\geq 36$ CAG-repeats) prodromal PREDICT-HD participants were included in the study (448 females and 267 males; ages 18-82 years; mean CAG-repeat number $=42.5$ $(\mathrm{SD}=2.5)$ ). Participant data included genotyping, T1-weighted structural MR images, cognitive and motor variables, and demographics (including age, sex, years of education, and CAG-repeat number) from PREDICT-HD [15]. Exclusion criteria included poor genomic or imaging data quality, relatedness to another participant, or the presence of any other central nervous system disorder or unstable medical or psychiatric condition. All PREDICT-HD participants provided written, informed consent and were treated in accordance with protocols approved by each participating institution's internal review board. Participants underwent genotyping before study enrolment, and those with more than 35 CAG-repeats who did not meet criteria for HD diagnosis were designated as prodromal.

\subsection{Data Availability}

SNP data included in this study are publicly available from dbGAP (Study Accession: phs000222.v4.p2). Other PREDICT-HD data, including baseline T1-weighted MR images (used as input files in this study), subcortical and cortical segmentations, and longitudinal clinical measurements, are available on the public download site (ftp:/ / ftp.ncbi.nlm.nih.gov / dbgap/studies / phs000222). Release of the specific genetic and imaging component data generated in this study is forthcoming; however, similar ICA-generated gray matter imaging components from a different PREDICT-HD study by the lead author have been made available in the latest study version (Study Accession: phs000222.v5.p2; Dataset Name: SBM_sMRI; Dataset Accession: pht006857.v1.p2). Access to the specific component data from the study may be requested from the corresponding author.

\subsection{Cognitive and Motor Variables}

Seven clinical variables were selected and tested for brain structural and genetic associations (outlined below), based on their established clinical reliability and sensitivity to prodromal HD progression [16]. Because we were interested in genetic and brain-structural effects on cognitive and motor functioning, we analyzed the portions of the Unified Huntington's Disease Rating Scale (UHDRS) relevant to these domains. The UHDRS includes four sections measuring movement, cognition, behavior, and functional capacity. We assessed the Total Motor Score (UHDRS-TMS), which comprises the movement portion of the UHDRS and is a sum of the scores on the individual motor variables (oculo-motor function, dysarthria, chorea, dystonia, gait, and postural stability). We also analyzed the Symbol Digit Modalities Test (SDMT) and the Stroop test (color, word, and interference conditions), which are two of the three parts of the UHDRS cognition section (we did not assess verbal fluency). In addition to the UHDRS-TMS, SDMT, and Stroop Interference, we also analyzed Trail-Making Tests A and B (TMTA and TMTB), which are not part of the UHDRS. We chose these tests because of our specific interest in cognition and motor functioning, and because previous work by the PREDICT-HD group demonstrated that these measures are particularly sensitive to prodromal changes in brain structure [16].

Briefly, the SDMT measures working memory, complex scanning, and processing speed, and is an adaptation of the Wechsler Digit Symbol subtest $[17,18]$. Participants are provided with a key of symbols paired with numbers at the top of the test page. On the same test sheet, a series of numbers are presented in a horizontal row, and the task is to fill in the symbol matching each number in the sequence as quickly and accurately as possible. Raw scores represent the number of correctly completed items within 90 s; thus, higher scores indicate better performance [19].

The Stroop Color and Word Test consists of three 45-s conditions that measure basic attention and inhibition of an overlearned response [20,21]. For the color condition, the task is to identify colors presented on stimulus cards. For the word condition, the task is to read color names presented in 
black ink. Both the color and word conditions measure basic attention. For the interference condition, participants identify the ink color in which color-names are printed, rather than reading the color name itself. For example, for the word "blue" printed in green ink, the correct response is "green." The interference condition measures the ability to inhibit the dominant (or automatic processing) response, which is to read the word. For each of the three conditions, raw scores reflect the number of correct trial responses, and higher scores thus reflect better performance.

TMTA and TMTB measure visual attention and task switching [22,23]. For TMTA, the task is to sequentially connect a series of numbered circles (e.g., 1-2-3-4, etc.) as quickly as possible. For TMTB, participants consecutively connect numbers and letters in ascending/alphabetical order, alternating between numbers and letters (e.g., 1-A-2-B-3-C, etc.). For both TMTA and TMTB, raw scores reflect the time (in seconds) taken to complete the task. Thus, higher scores reflect poorer performance.

The UHDRS-TMS assesses several indicators of motor performance spanning oculomotor function, bradykinesia, chorea, dystonia, gait, and postural stability [1,24]. Higher scores indicate poorer motor functioning.

\subsection{Genomic Data Preprocessing}

Data for 1,160,231 SNPs assayed with the Illumina Human 1M platform were downloaded from dbGAP (Study Accession: phs000222.v4.p2). Data were filtered at a 5\% missingness rate per sample and per SNP, and SNPs with a minor allele frequency (MAF) of $>0.05$ were selected. After linkage disequilibrium (LD) pruning $\left(r^{2}>0.5\right)$, the full genomic dataset consisted of 305,271 SNPs that were included in further analyses. Only one participant per family was included in the study; the family structure was determined using PLINK identity-by-descent analysis, where $p>0.18$ indicates relatedness (http:/ / zzz.bwh.harvard.edu/plink/ibdibs.shtml). The top 10 multi-dimensional scaling (MDS) factors were used to correct for population structure.

\subsection{Imaging Data Collection}

High-resolution anatomical MR images were collected at 33 sites using General Electric, Phillips, and Siemens scanners with field strengths of $1.5 \mathrm{~T}$ (Tesla) or $3 \mathrm{~T}$. The study used a standardized acquisition protocol that was modified for each site by our MR physicist. Secondary to upgrades and acquisition changes over the 12-year study, additional variation occurred. A total of 50 site/scanner field strength combinations were analyzed, with at least three participants from each different MRI scanner. Images at each site were obtained using three-dimensional (3D) T1-weighted inversion recovery turboflash (MP-RAGE) sequences. Then, $1.5 \mathrm{~T}$ scans were collected using General Electric and Siemens scanners. The Siemens protocol was constructed to be similar to the General Electric scan parameters: GRAPPA factor, $900 \mathrm{~ms}$ TI (inversion time), $2530 \mathrm{~ms}$ TR (relaxation time), $3.09 \mathrm{~ms}$ TE (excitation time), $256 \mathrm{~mm} \times 256 \mathrm{~mm}$ field of view (FoV), $10^{\circ}$ flip angle, 240 coronal slices with $1 \mathrm{~mm}$ slice thickness, $256 \times 128$ matrix with $1 / 4$ phase FoV, $220 \mathrm{~Hz} /$ pixel receiver bandwidth. Protocol for $3 \mathrm{~T}$ scanners commonly involved a sagittal localizing series followed by acquisition of an axial three-dimensional (3D) volumetric spoiled gradient recalled acquisition in a steady-state (GRASS) sequence, using the following scan parameters: $\sim 1 \mathrm{~mm} \times 1 \mathrm{~mm} \times 1.5 \mathrm{~mm}$ voxel size, $18 \mathrm{~ms}$ TR, $3 \mathrm{~ms}$ $\mathrm{TE}, 24 \mathrm{~cm} \mathrm{FoV}, 20^{\circ}$ flip angle, 124 slices with $1.5 \mathrm{~mm}$ slice thickness, $0 \mathrm{~mm}$ gap, $256 \times 192$ matrix with $3 / 4$ phase FoV, number of excitations $(\mathrm{NEX})=2$.

\subsection{Imaging Data Preprocessing}

Images were aligned with the anterior commissure-posterior commissure (AC-PC) plane, resampled with $1 \mathrm{~mm}$ isotropic voxels to correct for inhomogeneity [25], and preprocessed using SPM8 (http:/ / www.fil.ion.ucl.ac.uk/spm/software/spm8/). Images were segmented into gray matter, unmodulated (to isolate GM concentration), and normalized to the same SPM8 Montreal Neurological Institute (MNI) template. Voxels were re-sliced to $2 \times 2 \times 2 \mathrm{~mm}^{3}$, and images were smoothed by a $10 \times 10 \times 10 \mathrm{~mm}^{3}$ full-width-half-maximum (FWHM) Gaussian kernel. Processed images were 
$90 \times 109 \times 91$ voxels in size. A linear regression model was applied to each GM voxel to account for the effects of age, sex, and site (inclusive of field strength); the site variable was coded using 49 dummy variables.

\subsection{Parallel ICA with Reference (}

Prodromal GMC and SNP patterns were identified and tested for correlations by applying pICAr to the GMC imaging and the genomic data. SNPs within nine genes implicated in BDNF-signaling were included as references: BDNF, NGFR, NTRK2, RCOR1, SIN3A, SORT1, HAP1, REST, and RILP (see Table 1). SNPs within $20 \mathrm{kbp}$ of these genes were also included to capture regulatory elements in intronic and intergenic regions.

Table 1. Genes from which available single nucleotide polymorphisms (SNPs) were included as references for parallel independent component analysis with reference (pICAr).

\begin{tabular}{|c|c|c|c|c|}
\hline Factor & Gene(s) & $\begin{array}{l}\text { pICA Reference } \\
\text { SNPs (\#) }\end{array}$ & Full Name & Function \\
\hline REST/NRSF & $\begin{array}{c}\text { REST, } \\
\text { RCOR1, } \\
\text { RCOR3 }^{+}\end{array}$ & 4 & $\begin{array}{l}\text { RE1 silencing transcription } \\
\text { factor/neuron-restrictive } \\
\text { silencer factor }\end{array}$ & Transcriptional repression \\
\hline $\operatorname{Sin} 3 \mathrm{~A}$ & $\operatorname{SIN} 3 A$ & 2 & $\begin{array}{l}\text { SIN3 transcription regulator family } \\
\text { member A }\end{array}$ & $\begin{array}{c}\text { Part of co-repressor complex with } \\
\text { REST and coREST }\end{array}$ \\
\hline CoREST & RCOR1 & 9 & REST co-repressor & $\begin{array}{c}\text { Part of co-repressor complex with } \\
\text { REST and Sin3A }\end{array}$ \\
\hline HAP1 & HAP1 & 7 & Huntingtin-associated protein 1 & $\begin{array}{l}\text { Binds to huntingtin, facilitates } \\
\text { brain-derived neurotrophic factor } \\
\text { (BDNF) transcription and transport }\end{array}$ \\
\hline TrkB & NTRK2 & 52 & Tropomyosin receptor kinase B & BDNF high-affinity receptor \\
\hline P75 & NGFR & 26 & $\begin{array}{l}\text { Low-affinity nerve growth } \\
\text { factor receptor }\end{array}$ & BDNF low-affinity receptor \\
\hline RILP & RILP & 5 & REST-interacting LIM domain protein & REST nuclear receptor \\
\hline Sortilin & SORT1 & 12 & Sortilin 1 & $\begin{array}{l}\text { Suggested apoptotic function with } \\
\text { p75 and pro-BDNF }\end{array}$ \\
\hline BDNF & $B D N F$ & 6 & Brain-derived neurotrophic factor & $\begin{array}{l}\text { Neuronal growth, survival, } \\
\text { differentiation }\end{array}$ \\
\hline
\end{tabular}

PICA is an extension of ICA, a robust and popular method for isolating maximally independent sources from a mixed signal $[10,11,26]$. In a general ICA model, $X=A S: X$ is an observation (i.e., subject-by-variable matrix); $\mathrm{S}$ is a statistically independent component matrix (component-by-variables); and A is the loading coefficient or mixing matrix, the representation of each component in the subject or sample (subject-by-component). PICA performs this extraction simultaneously on two modalities, $\mathrm{X} 1$ and $\mathrm{X} 2$. For this experiment, $\mathrm{X} 1$ was a participants-by-voxels matrix of the masked GMC images, and X2 was a participants-by-loci matrix of the 305,271 SNPs. pICAr decomposes the observation into maximally independent sources (component matrices $\mathrm{S} 1$ and S2) by updating W1 and W2 (unmixing matrices) to optimize $\mathrm{F}_{1}$ (Infomax algorithm that maximizes the independence of modality- 1 components), $\mathrm{F}_{2}$ (modified Infomax that optimizes the modality- 2 component independence AND similarity to the reference matrix), and $\mathrm{F}_{3}$ (maximizes the correlations between the two modalities' loading coefficient matrices, A1 and A2) [10,11]. Each reference is a vector containing alleles of a gene likely to be in linkage disequilibrium, meaning they co-occur more or less frequently than expected for independent loci. A reference vector for each gene listed in Table 1 comprises the reference matrix r. pICA outputs a loading coefficient for each participant for each SNP and imaging profile, representing how much the participant's genome and brain structure matches each profile detected in the sample. 


$$
\begin{gathered}
X_{d}=A_{d} S_{d} \rightarrow S_{d}=W_{d} X_{d}, A_{d}=W_{d}^{-1}, d=1,2 \\
Y_{d}=\frac{1}{1+e^{-u_{d}}}, U_{d}=W_{d} X_{d}+W_{d 0} \\
F_{1}=\max \left\{H\left(Y_{1}\right)\right\}=\max \left\{-E\left[\ln f_{y_{1}}\left(Y_{1}\right)\right]\right\} \\
F_{2}=\max \left\{\lambda H\left(Y_{2}\right)+(1-\lambda)\left[-\operatorname{dist}^{2}\left(\widetilde{r}_{,}\left|\widetilde{S}_{2 k}\right|\right)\right]\right\} \\
=\max \left\{\lambda\left(-E\left[\ln f_{y 2}\left(Y_{2}\right)\right]\right)+(1-\lambda)\left(-\left\|\left|W_{2 k} \widetilde{X}_{2}\right|-\widetilde{r}\right\|_{2}^{2}\right)\right\} \\
F_{3}=\max \left\{\sum_{i, j} \operatorname{Corr}^{2}\left(A_{1 i}, A_{2 i}\right)\right\}=\max \left\{\sum_{i, j} \frac{\operatorname{Cov}^{2}\left(A_{1 i}, A_{2 i}\right)}{\operatorname{Var}\left(A_{1 i}\right) \operatorname{Var}\left(A_{2 i}\right)}\right\}
\end{gathered}
$$

\subsection{SNP and GMC Correlations with Clinical Variables}

We then queried SNPs of interest (i.e., SNPs within Table 1 BDNF-signaling genes that were also highlighted in the pICA results) for correlations with the cognitive and motor variables. Five SNPs in NTRK2 were tested in separate multivariate general linear model (GLM) tests using SPSS [27]; the clinical measures were dependent variables, and the SNP value (a continuous variable between 0 and 2) was a covariate. We similarly examined associations between the clinical variables and a pICA SNP and GMC component that were significantly correlated with each other. Here, the clinical measures were the dependent variables and the SNP or GMC loading coefficient was a covariate.

\subsection{Associations of Top-Weighted Component SNPs with Clinical Variables}

To test whether the observed effects were explained by the entire component cumulatively or were largely the effect of heavily-weighted SNPs, restricted genetic coefficients based on the most important SNPs in the component were calculated. First, the distribution of the weights for the 305,271 SNPs in the SNP component was fitted to a logistic distribution. Based on this, SNPs with weights more than 4.25 standard deviations (SDs) from the mean were selected as top contributing SNPs $(N=61)$. Weights for these 61 SNPs within the component $(61 \times 1$ matrix $)$ were multiplied by participant genotypes for these SNPs $(715 \times 61$ matrix $)$ to yield new participant loadings for the top 61 contributing SNPs. These new loadings were correlated with loadings for the full component at $r(713)=0.77$ (two-tailed significance of $p<0.001$ ). A list of the top $61 \mathrm{SNPs}$, associated genes, and weights in the component is available in Supplementary Materials Table S1.

\subsection{Confirmation of Significant Results}

Permutation testing and leave-N-subjects-out (10-fold, $10 \%$ of total sample) cross-validation were used to verify significant pICA component correlations $[10,28]$. Permutation testing involves a random shuffling/mismatching of participant SNP and GMC data, which are then subjected to pICA and correlation testing. The permutation testing is performed multiple (in this case, 1000) times, allowing a null distribution to be formed that reveals the likelihood of obtaining the significant GMC-SNP correlation by chance. Leave-N-out (or 10-fold validation) is achieved by running pICA on 10 separate datasets, each containing $90 \%$ of the full data, to determine if the results from the original analysis are replicable.

\subsection{Regression Influence Plot}

An influence matrix was generated using the regression influence plot function in $\mathrm{R}[29,30]$ to pinpoint any individuals disproportionately driving the outcome of the significant GMC-SNP component correlation. This function outputs a plot of studentized residuals by hat values as well as a data frame containing hat values, studentized residuals, and Cook's distances. The approach efficiently describes the influence that each dependent variable value has on each fitted or predicted value. 


\section{Results}

\section{1. $p I C A r$}

In our PREDICT-HD prodromal cohort, pICAr detected a significantly correlated GMC-SNP component pair $(\mathrm{r}(713)=0.17, p<0.001)$. This association remained significant after Bonferroni correction for multiple testing. The GMC component most strongly represented supplementary motor, superior, mid, and medial frontal, and precentral/primary motor regions (Figure 2). The correlated SNP component had strong contributions from the NTRK2 gene, which encodes BDNF's high-affinity receptor type (TrkB) (Figure 3). Four intronic NTRK2 SNPs (rs11140810, rs4877289, rs10868241, rs2277193) were among the top 10 SNPs contributing to the component. Other top genes included CDK14, FAM114A1, and HEATR4 (Table 2).

a)
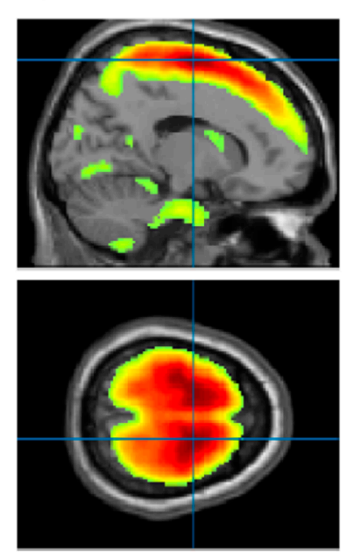
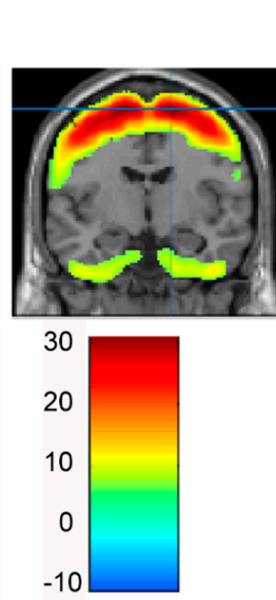

b)

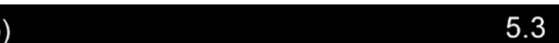

5.3

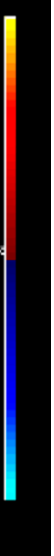

Figure 2. The frontal gray matter concentration (GMC) component significantly paired with the NTRK2 SNP component in pICAr: (a) maximum effects in right premotor and supplementary motor. Crosshairs are positioned at the global maximum $(\mathrm{T}(1)=30.75)$ and reach a threshold at $p=0.05$; (b) multi-slice axial topography (threshold: $Z=2.5$ ) showing a mostly positive component with strong representation from superior frontal gray matter and Brodmann area 6 (supplementary and premotor cortex). Images are displayed using xjView ( $\mathrm{a}$; http://www.alivelearn.net/xjview) and Fusion ICA Toolbox (b; http:/ / mialab.mrn.org/software/fit).

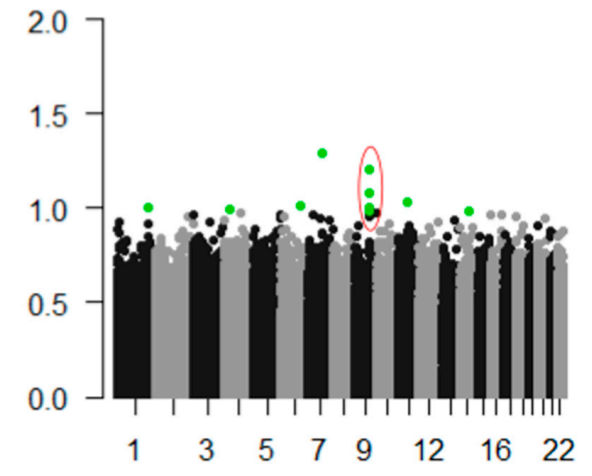

Chromosome

Figure 3. Manhattan plot showing top-weighted SNPs within the SNP component that correlated with a frontal/supplementary motor gray matter profile in parallel ICA. The top 10 SNPs contributing to the component are highlighted in green, and the four top NTRK2 SNPs are circled in red. The $Y$ axis indicates each SNP's weight, or contribution, to the SNP component. The plot was generated using the qqman package in $\mathrm{R}$ version 3.4.1. 
Table 2. The 10 SNPs most heavily weighted in the SNP component, with reference SNP cluster IDs (rs IDs), weights in the component and their directions, associated genes, minor allele frequencies, and variant class. SNV = single nucleotide variation, DIV = deletion/insertion variation.

\begin{tabular}{|c|c|c|c|c|c|c|}
\hline SNP & Weight (I I) & $+1-$ & Ranking & Gene & Minor Allele Frequency & Class \\
\hline rs7801922 & 1.29 & + & 1 & CDK14 & $\begin{array}{c}\mathrm{T}=0.34 / 1704(1000 \text { Genomes }) \\
\mathrm{T}=0.38 / 11000 \text { (TOPMED) }\end{array}$ & SNV \\
\hline rs11140810 & 1.20 & + & 2 & NTRK2 & $\begin{array}{c}\mathrm{G}=0.42 / 2104(1000 \text { Genomes }) \\
\mathrm{G}=0.42 / 12329(\text { TOPMED })\end{array}$ & SNV \\
\hline rs4877289 & 1.08 & + & 3 & NTRK2 & $\begin{array}{c}\mathrm{G}=0.38 / 1926(1000 \text { Genomes }) \\
\mathrm{G}=0.38 / 11160 \text { (TOPMED) }\end{array}$ & SNV \\
\hline rs548321 & 1.03 & + & 4 & $70 \mathrm{~kb} 5^{\prime}$ of $L R R C 55$ & $\begin{array}{c}\mathrm{G}=0.41 / 2055(1000 \text { Genomes }) \\
\mathrm{G}=0.38 / 11140(\text { TOPMED })\end{array}$ & SNV \\
\hline rs112140519 & 1.01 & + & 5 & $53 \mathrm{~kb} \mathrm{3}$ of NUS1 & $-=0.33 / 1652$ (1000 Genomes) & DIV \\
\hline rs427790 & 1.00 & + & 6 & $\begin{array}{l}\text { MIR181A1, } \\
\text { NR5A2 }\end{array}$ & $\begin{array}{c}\mathrm{C}=0.33 / 1658(1000 \text { Genomes }) \\
\mathrm{C}=0.38 / 10948(\mathrm{TOPMED})\end{array}$ & SNV \\
\hline rs10868241 & 1.0 & + & 7 & NTRK2 & $\begin{array}{c}\mathrm{A}=0.32 / 1614(1000 \text { Genomes }) \\
\mathrm{A}=0.24 / 6986(\mathrm{TOPMED})\end{array}$ & SNV \\
\hline rs7655305 & 0.99 & + & 8 & FAM114A1 & $\begin{array}{c}\mathrm{G}=0.43 / 2140(1000 \text { Genomes }) \\
\mathrm{G}=0.43 / 12519(\text { TOPMED })\end{array}$ & SNV \\
\hline rs2277193 & 0.98 & + & 9 & NTRK2 & $\begin{array}{c}C=0.34 / 1679(1000 \text { Genomes }) \\
C=0.41 / 11827(\text { TOPMED })\end{array}$ & SNV \\
\hline rs8012614 & 0.98 & + & 10 & HEATR4 & $\begin{array}{c}C=0.29 / 1442(1000 \text { Genomes }) \\
C=0.37 / 10860(\text { TOPMED })\end{array}$ & SNV \\
\hline
\end{tabular}

\subsection{SNP and GMC Correlations with Clinical Variables}

The frontal GMC component highlighted by pICA (and associated with the NTRK2 SNP component) was significantly correlated with four of the seven queried clinical variables, including UHDRS total motor score $(\mathrm{F}(1,672)=6.8, p=0.009$, surviving Bonferroni multiple testing correction), TMTA $(\mathrm{F}(1,672)=6.3, p=0.01$, passing Bonferroni), Stroop Color $(\mathrm{F}(1,672)=4.7, p=0.03)$, and Stroop Interference $(\mathrm{F}(1,672)=3.8, p=0.05)$. This component also approached a significant correlation with the other three clinical variables (SDMT: $\mathrm{F}(1,672)=3.5, p=0.06$; Stroop Total: $\mathrm{F}(1,672)=3.4, p=0.07$; TMTB: $F(1,672)=3.1, p=0.08)$ ). In each case, a higher GMC was linked to improved performance.

The frontal-GMC-associated NTRK2 SNP component was not significantly related to the clinical measures. However, one of the top NTRK2 SNPs contributing to the component (rs2277193) was significantly associated with TMTB $(\mathrm{F}(1,672)=6.7, p=0.01)$, an effect that survived Bonferroni correction. Here, the minor allele was associated with better TMTB performance. This SNP also approached a significant correlation with Stroop Interference $(\mathrm{F}(1,672)=3.6, p=0.058)$, with the minor allele similarly being associated with better performance. NTRK2 SNP rs111408010 was also correlated with UHDRS-TMS $(\mathrm{F}(1,672)=3.7, p=0.05)$, with a greater minor allele number being associated with better functioning (lower TMS score), but this effect did not withstand multiple testing correction.

\subsection{Confirmation of Significant Results}

The pICA results passed permutation testing; the ratio of correlation values above maximally linked components was 0.013 , translating to about a $1 \%$ likelihood that the results were obtained by chance. However, the results did not replicate in all the 10-fold validation runs, likely because the GMC-SNP component correlation was driven by several individuals with high or low levels of the SNP component (Figure 4). The visual interpretation was confirmed by the regression influence analysis, which highlighted 25 participants that dominated the association (Supplementary Materials Figure S1). All of these participants had SNP component values 2-4 SDs above or below the mean. 

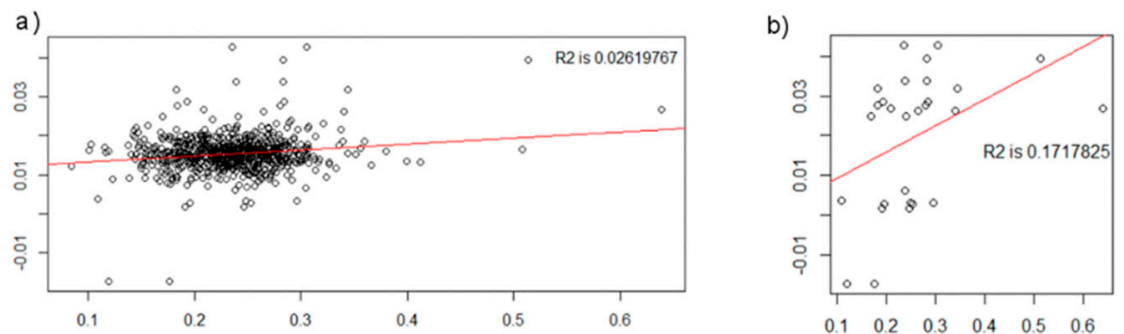

Figure 4. Correlation between pICA frontal GMC and NTRK2 SNP components in (a) the full prodromal sample and (b) participants with SNP component values $\geq 2$ SDs above or below the mean $(N=28)$. Mean SNP component value $=0.015$ (full prodromal sample), SD $=0.005, X$ axis $=$ SNP component weights, $Y$ axis $=$ GMC component weights, $\mathrm{R} 2=r^{2}$. Graphics generated with R version 3.4.1.

\subsection{Associations of Top-Weighted Component SNPs with Clinical Variables}

Similar to tests with the full SNP component, clinical associations with weights comprised of the top 61 component SNPs yielded no significant results. Derived $p$ and $r^{2}$ values were slightly weaker for the top 61 weights but were essentially comparable.

\section{Discussion}

\subsection{High or Low Levels of the NTRK2 SNP Profile Affect Prodromal Frontal GMC}

Our results suggest that a strong or weak presence of the NTRK2-weighted genetic profile may be required to significantly affect the frontal gray matter profile. The genetic effect on frontal/supplementary motor GMC in individuals in the tail of the sample was still strong enough to be significant and apparent within the larger sample. In keeping with this, all 25 individuals driving the significant SNP-GMC component relationship (according to the regression influence analysis) had SNP component scores 2-4 SDs above or below the mean; only three individuals with SNP component values in this range were not highlighted by the regression influence test. By contrast, only six of these participants also had GMC component values $\geq 2$ SDs from the mean in either direction, suggesting that the effect is specific to the SNP component. These participants did not stand out in any other obvious way from the full prodromal sample, and represented both sexes and a variety of ages, sites, and CAG-repeat numbers.

Four NTRK2 SNPs were among the top 10 SNPs contributing to the GMC-related SNP component. NTRK2 (Neurotrophic Receptor Tyrosine Kinase 2) is a large (358,613 base) chromosome 9 gene located between SLC28A3 (solute carrier family 28, sodium-coupled nucleoside transporter member 3) and AGTPBP1 (ATP/GTP binding protein 1). NTRK2 is widely expressed in the brain (Figure 5) and encodes BDNF's high-affinity TrkB (tropomyosin receptor kinase B) receptor. TrkB has important cellular functions that may underlie NTRK2's prominence and protective role in this study. 


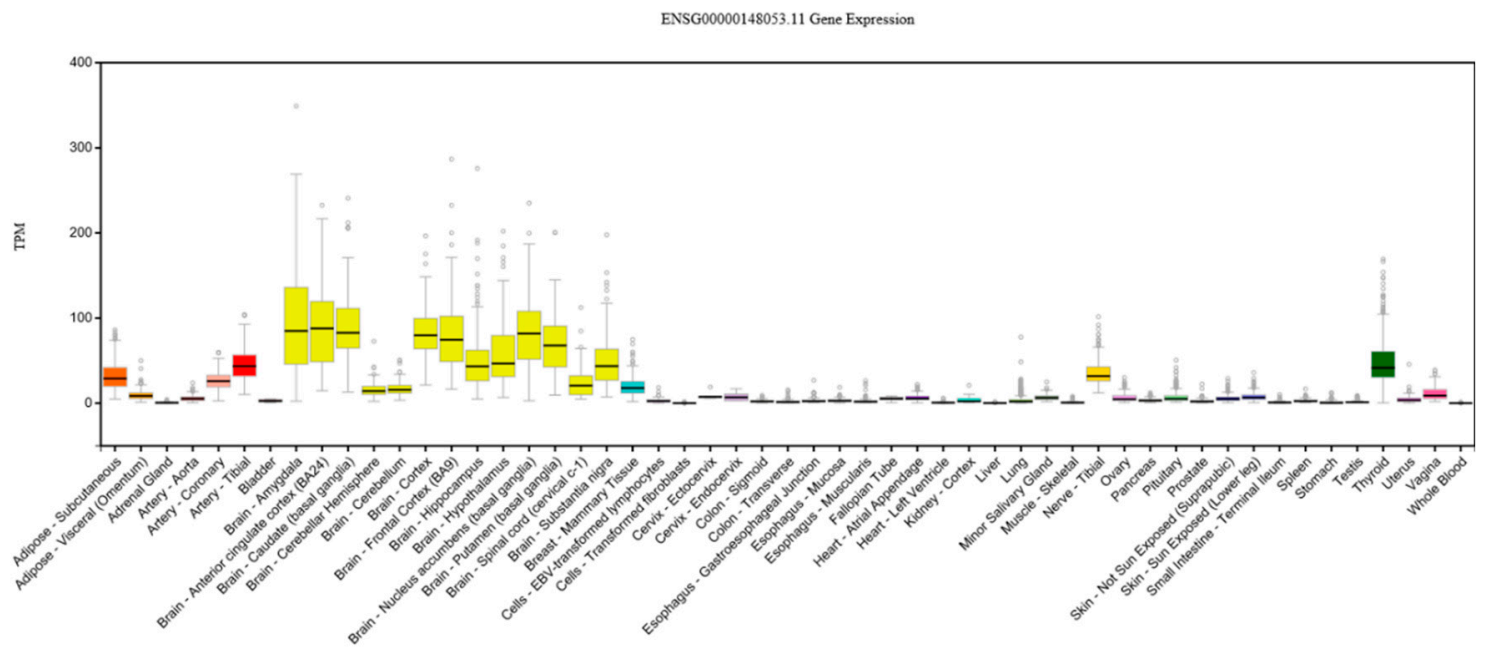

Figure 5. Expression of NTRK2 in various tissue types, showing relatively strong expression in brain tissue. TPM = transcripts per kilobase million. Expression threshold: $>0.1$ TPM and $\geq 6$ reads in $20 \%$ or more of samples. Box plots are 25th and 85th percentiles, with a black line at the median. Outliers are \pm 1.5 times the interquartile range. Data source: GTEx Analysis Release V7 (dbGaP Accession phs000424.v7.p2).

TrkB binds with BDNF, neurotrophin-4 (NTF4), and neurotrophin-3 (NTF3), and regulates neuronal differentiation, growth, and survival as well as synaptic plasticity and the transcription of cell survival genes [31]. Thus, it affects both long- and short-term learning and memory by mediating short-term synaptic function as well as long-term potentiation. TrkB has also been implicated in apoptosis-suppression and the promotion of communication between neurons and glial cells.

Regarding BDNF, TrkB is preferentially activated by mature BDNF and expressed in indirect pathway striatal MSNs [9]. Following activity-dependent, anterograde transport by cortical afferents, BDNF can act post-synaptically on TrkB receptors to inhibit GABAergic or enhance glutamatergic synaptic transmission. By interacting with presynaptic TrkB receptors, BDNF can also be retrogradely transported to the cell body, where it stimulates cortical glutamate release. TrkB also responds to neuronal damage; its mRNA increases following excitotoxic lesions, and reduced TrkB is associated with neurodegeneration in Alzheimer's disease. In the context of HD, mHTT reduces TrkB expression in a CAG-dependent manner (in R6/1 mice), an effect that is rescued by mHTT inactivation [9].

Like other neurotrophins, BDNF is synthesized in the endoplasmic reticulum (ER) as a large (32 $\mathrm{kDa}$ ) precursor protein (pro-BDNF) [9], then translocated to the Golgi complex and secretory vesicles, proteolytically cleaved, and released as mature BDNF (14 kDa). P75, BDNF's low-affinity receptor (encoded by NGFR), is preferentially activated by pro-BDNF and promotes apoptosis. There is evidence for a cortical pro-BDNF overabundance in $\mathrm{HD}$, which is one explanation for the lack of consensus on cortical BDNF reductions in HD and the prodrome [9]; pro-BDNF increases could mask BDNF reductions when probed with methods that do not differentiate between pro- and mature BDNF. In humans, a highly conserved and fairly common (20-30\% heterozygotes, $\sim 4 \%$ homozygotes) BDNF polymorphism (Val66Met) in the $5^{\prime}$ region that encodes pro-BDNF is associated with memory deficits and multiple disorders [14]. Mature BDNF production is not affected by Val66Met, but pro-BDNF trafficking and packaging are substantially impacted. Interestingly, mHTT significantly blocks the post-Golgi trafficking of Val66Val (but not Val66Met) BDNF, although neither allele is associated with disrupted transport from the ER to the Golgi.

Considering the roles of BDNF's high- and low-affinity receptor types in promoting cell survival and apoptosis, respectively, a pro- to mature BDNF ratio imbalance could contribute to the abnormal apoptosis observed in HD [14]. TrkB receptor underrepresentation could hinder BDNF's ability to increase its own expression. Similarly, p75 receptor or pro-BDNF overabundance could amplify 
apoptosis and contribute to disease-related decline. The results of the present study suggest that NTRK2 variations could influence this balance protectively, promoting cell survival and preserving frontal gray matter and cognitive and motor performance.

\subsection{The SNP-GMC Component Correlation is Not an Aggregate Effect of the Entire SNP Component}

Weights comprised of the top 61 SNPs contributing to the component (rather than the full component with weights for all 305,271 SNPs) yielded comparable results when evaluated with the GMC profile and clinical variables. Derived $p$ and $\mathrm{r}^{2}$ values were only slightly weaker in analyses using the top 61 SNP weights, indicating that the original results do not reflect the aggregate effects of the entire SNP component but are rather primarily due to the top contributing SNPs.

\subsection{The NTRK2-Associated Frontal Gray Matter Profile Is Related to Prodromal Cognitive and Motor Functioning}

The correlated GMC component, containing supplementary motor, superior, middle, and medial frontal, and precentral/primary motor cortex, was significantly related to both cognitive (TMTA, Stroop Color, Stroop Interference) and motor (TMS) performance in this cohort. Although the variance in individual clinical performance measures accounted for by the GMC component was small, such effects are typical when comparing single clinical variables to brain structural measures; the observed associations were consistent with the literature reporting structural and functional changes in these regions that correlate with altered performance on these tasks in independent samples [32-36]. Although each of the cognitive tasks has a motor component (writing or verbalization is required for response), the stronger correlation for TMS compared to the cognitive measures likely reflects the strength of the supplementary and primary motor cortex in the GMC component and the increased demand on these regions for motor relative to cognitive functioning [37]. This may also explain why significant effects were not observed for SDMT and Stroop Word, as motor involvement in these tasks is relatively limited.

\subsection{Top Contributing NTRK2 SNPS}

Among the top 10 SNPs contributing to the frontally-related SNP component were rs11140810, rs4877289, rs10868241, and rs2277193; no other gene was represented more than once in the top 10 SNPs. Interestingly, three of these four SNPs are between NTRK2 exons 19 and 20 (the location of an alternative stop codon), with rs2277193 (the TMTB-related SNP) being the closest to exon 19. In fact, rs2277193 is only 799 base pairs from rs 11140810 , the strongest NTRK2 contributor to the component, and it is 3577 base pairs from the other top SNP in this region (rs10868241). The placement of these SNPs (as well as the fourth NTRK2 SNP, located between exons 16 and 17) is fitting, as truncated NTRK2 isoforms that lack the catalytic tyrosine kinase domain (generated by alternate terminal exon 16 or exon 19) are considered the most clinically relevant [38].

Of the NTRK2 SNPs, rs11140810 had the strongest weight in the component. The Lieber Institute for Brain Development's (LIBD) expression quantitative trait loci (eQTL) browser, comprised of dorsolateral prefrontal cortex (DLPFC) expression data from 237 healthy controls and 175 schizophrenia patients, identified this SNP as an eQTL for NTRK2 in the DLPFC at the expressed region level [39]. According to Braineac (http://www.braineac.org), an eQTL database from 134 healthy human brains [40], the regulatory effects of rs 11140810 on NTRK2 are strongest in the occipital lobe, thalamus, and frontal cortex. Other top affected genes include SLC28A3, HNRNPK, MIR7-1, and NAA35. Table 3 lists the top component SNPs and their strongest reported regulatory effects on genes in the brain. HaploReg, a tool for visualizing 1000 Genomes Project SNP LD information in conjunction with Roadmap Epigenomics and ENCODE chromatin state and protein binding annotation, shows an association of rs11140810 with markers of H3K27 acetylation (linked to active transcription) in the brain (Table 4), as well as altered regulatory motifs including forkhead box (Foxa), glioma-associated oncogene (GLI), hypermethylated in cancer (Hic1), and zinc finger protein (Zec) [41]. 
The nearby rs2277193 had a protective effect on TMTB, in which the minor allele was associated with improved performance. In the brain, this SNP exhibits maximum regulatory effects on many of the same genes, albeit a less pronounced influence on NTRK2 [40]. According to Haploreg, this SNP is associated with expression-enhancing chromatin states in the brain (Table 4), altered glucocorticoid receptor (GR) and paired-box protein (Pax-6) regulatory motifs, and AGTPBP1 expression in the prefrontal cortex (PFC) [41].

rs4877289 is correlated with H3K4me1 (enhancer activity associated) in anterior caudate and cingulate gyrus, and is in strong LD with other NTRK2 SNPs associated with histone enhancement and promotion, protein binding, altered regulatory motifs, and eQTL hits. For example, rs 11140785 $(\mathrm{LD}=0.94)$ bound to STAT3 in ChIP-Seq and is associated with altered myocyte enhancer factor (Mef2) regulatory motif and expression-enhancing chromatin changes in the hippocampus, substantia nigra, caudate, inferior temporal, cingulate gyrus, angular gyrus, and DLPFC. In the brain, this variant most strongly affects RMI1, especially in the frontal cortex [40].

Like rs11140810, rs10868241 is an identified eQTL for NTRK2 in the DLPFC at the expressed region level in control and disease populations [39]. It is also associated with enhancer and promoter-associated active histone modifications in several brain regions (Table 4), and is in strong LD with SNPs linked to enhancer histone marks and altered regulatory motifs (for example, rs4457413 $\left(\mathrm{LD} r^{2}=0.97\right)$ is associated with alteration of 12 regulatory motifs and with histone enhancers in the temporal lobe and PFC). In the brain, rs10868241 most strongly regulates many of the same genes as the other top NTRK2 SNPs (SLC28A3, HNRNPK, MIR7-1, NTRK2, AGTPBP1, RMI1) [40], especially in the frontal cortex and hippocampus. 
Table 3. Top SNPs contributing to supplementary/frontal-related pICA SNP components and reported effects on gene expression in the brain. These SNPs are associated with gene expression changes most prominently in the frontal lobe (in agreement with the study results), thalamus, putamen, and cerebellum. The first column lists the SNP rs ID, associated or closest gene, and gene type $(\mathrm{I}=$ intronic, $\mathrm{IG}=$ intergenic). Occ. = occipital, Thal. $=$ thalamus, Temp. $=$ temporal, $\mathrm{WM}=$ white matter, Put = putamen, Hipp. = hippocampus, Fron. = frontal, Cereb. = cerebellum, SNigra $=$ substantia nigra, Med. $=$ medulla, DLPFC $=$ dorsolateral prefrontal cortex. ${ }^{\dagger}=$ Genes associated with more than one SNP and more than tissue type; ${ }^{*}=$ Genes associated with more than one tissue type. Expression data was obtained from Braineac [40]

\begin{tabular}{|c|c|c|c|c|c|c|c|c|c|c|c|}
\hline SNP (rs) & Occ. & Thal. & Temp. & WM & Put. & Hipp. & Fron. & Cereb. & SNigra & Med. & DLPFC \\
\hline $\begin{array}{c}8012614 \\
\text { HEATR4 (I) }\end{array}$ & $\begin{array}{c}\text { NUMB; } \\
\text { TMEM90A }\end{array}$ & HEATR4* & & & ACOT4* & $\begin{array}{l}\text { RBM } 25^{*} ; \\
\text { ACOT4 * }\end{array}$ & & RBM $25^{*}$ & RBM $25^{*}$ & & $\begin{array}{c}\text { RBM } 25^{*} ; \\
\text { ACOT4 *; } \\
\text { HEATR4 } \\
\text { DNAL1 }\end{array}$ \\
\hline $\begin{array}{c}7801922 \\
\text { CDK14 (I) }\end{array}$ & STEAP1 & $\begin{array}{l}\text { C7orf63; } \\
\text { STEAP2 }\end{array}$ & & STEAP2 ${ }^{*}$ & & C7orf6 $63^{*}$ & & STEAP2* & $\begin{array}{l}\text { DPY19L2P4; } \\
\text { CDK14* }\end{array}$ & & C7orf63* \\
\hline $\begin{array}{c}7655305 \\
\text { FAM114A1 (I) }\end{array}$ & & & RPL6 & PDS5A & & FAM114A1* & TLR6 & $\begin{array}{c}\text { PTTG2; } \\
\text { FLJ13197; } \\
\text { UGDH }\end{array}$ & FAM114A1 * & FAM114A1* & TLR1 \\
\hline $\begin{array}{c}548321 \\
\text { LRRC55 (IG) }\end{array}$ & & & & & $\begin{array}{l}\text { UBE2L6; } \\
\text { ZDHHC5 }\end{array}$ & & TIMM10 & & & & \\
\hline $\begin{array}{c}427790 \\
\text { MIR181A1, } \\
\text { NR5A2 (IG) }\end{array}$ & & NEK7 & $\begin{array}{c}\text { NR5A2 }{ }^{*} ; \\
\text { PTPRC; } \\
\text { ATP6V1G3 }\end{array}$ & & $\begin{array}{l}\text { NR5A2 }{ }^{*} \text {; } \\
\text { MIR181A1 }\end{array}$ & & MIR181A1* & MIR181A1* & $N R 5 A 2^{*}$ & & \\
\hline $\begin{array}{c}11140810 \\
\text { NTRK2 (I) }\end{array}$ & NTRK $2^{+}$ & $\begin{array}{l}\text { NTRK2 }{ }^{+} \\
\text {HNRNPK } \\
\text { MIR7- }^{+}\end{array}$ & & $\begin{array}{l}\text { NTRK2 }{ }^{+} \\
S L C 28 A 3^{\dagger}\end{array}$ & $N A A 35^{\dagger}$ & NTRK $2^{\dagger}$ & NTRK $2^{+}$ & $\begin{array}{l}\text { HNRNPK }{ }^{+} \\
\text {MIR7-1 }^{+}\end{array}$ & & $S L C 28 A 3^{+}$ & NTRK ${ }^{+}$ \\
\hline $\begin{array}{c}2277193 \\
\text { NTRK2 (I) }\end{array}$ & & $\begin{array}{l}\text { SLC28A3 }{ }^{+} \\
\text {HNRNPK } \\
\text { MIR7- }^{+}\end{array}$ & & $S L C 28 A 3^{\dagger}$ & & $N A A 35^{\dagger}$ & $\begin{array}{l}S L C 28 A 3^{\dagger} \\
A G T P B P 1^{\dagger}\end{array}$ & $\begin{array}{c}\text { SLC28A3 }^{+} ; \\
\text {HNRNPK }^{+} ; \\
\text {MIR7-1 } \\
\text { NAA35 ; } \\
\text { AGTPBP1 }^{+}\end{array}$ & $N A A 35^{\dagger}$ & $S L C 28 A 3^{+}$ & \\
\hline $\begin{array}{c}4877289 \\
\text { NTRK2 (I) }\end{array}$ & $A G T P B P 1^{+}$ & & & $\begin{array}{l}\text { HNRNPK }{ }^{+} \\
\text {MIR7-1 }^{+}\end{array}$ & $S L C 28 A 3^{\dagger}$ & & $\begin{array}{c}\text { RMI1 }^{+} \\
\text {SLC28A3 }^{+} \\
\text {AGTPBP }\end{array}$ & & & & \\
\hline $\begin{array}{c}10868241 \\
\text { NTRK2 (I) }\end{array}$ & & $\begin{array}{l}\text { HNRNPK }^{+} \\
\text {MIR7- }^{+}\end{array}$ & & $S L C 28 A 3^{\dagger}$ & RMI1 $^{+}$ & $S L C 28 A 3^{+}$ & $\begin{array}{l}\text { SLC28A3 }{ }^{+} \\
\text {NTRK }^{+}\end{array}$ & & & $\begin{array}{c}\text { HNRNPK }^{+} ; \\
\text {MIR7-1 } \\
\text { AGTPBP }^{+}\end{array}$ & NTRK ${ }^{+}$ \\
\hline
\end{tabular}


Table 4. Genes regulated by prominent NTRK2 SNPs in this study, along with their associated functions and pathways. Sources: GeneCards human gene database [31] and UniProt [42].

\begin{tabular}{|c|c|c|c|c|c|}
\hline Gene Name & Full Name & Associated NTRK2 SNP(s) & Description & Type & Related Pathways \\
\hline SLC28A3 & Solute Carrier Family 28 Member 3 & $\begin{array}{l}\text { rs1114081, rs2277193, } \\
\text { rs4877289, rs10868241 }\end{array}$ & $\begin{array}{l}\text { Neurotransmission, vascular tone, adenosine } \\
\text { concentration near cell surface receptors, } \\
\text { transport/metabolism of nucleoside drugs }\end{array}$ & $\begin{array}{l}\text { Protein coding, } \\
\text { nucleoside transporter }\end{array}$ & $\begin{array}{l}\text { Vitamin and nucleoside transport, } \\
\text { thiopurine pathway, } \\
\text { pharmacokinetics/pharmacodynamics }\end{array}$ \\
\hline AGTPBP1 & ATP/GTP Binding Protein 1 & rs10868241 & $\begin{array}{l}\text { Contains nuclear localization signals and an } \\
\text { ATP/GTP-binding motif, involved in the } \\
\text { deglutamylation of protein polyglutamate side chains, } \\
\text { removal of gene-encoded polyglutamates from } \\
\text { protein carboxy-terminus, and shortening of long } \\
\text { polyglutamate chains }\end{array}$ & $\begin{array}{l}\text { Protein coding, zinc } \\
\text { carboxypeptidase, } \\
\text { metallocarboxypeptidase }\end{array}$ & Neuroscience \\
\hline HNRNPK & $\begin{array}{l}\text { Heterogeneous Nuclear } \\
\text { Ribonucleoprotein K }\end{array}$ & $\begin{array}{l}\text { rs1114081, rs2277193, } \\
\text { rs4877289, rs10868241 }\end{array}$ & $\begin{array}{l}\text { Major pre-mRNA-binding protein, binds to poly(C) } \\
\text { sequences, involved in nuclear metabolism of } \\
\text { hnRAs, and p53/TP53 response to DNA damage } \\
\text { (transcriptional activation and repression) }\end{array}$ & $\begin{array}{l}\text { Protein coding, } \\
\text { heterogeneous nuclear } \\
\text { ribonucleoprotein } \\
\text { (hnRNP) }\end{array}$ & Translational control and mRNA splicing \\
\hline MIR7-1 & MicroRNA 7-1 & $\begin{array}{l}\text { rs1114081, rs2277193, } \\
\text { rs4877289, rs10868241 }\end{array}$ & Affiliated with an undefined RNA class & RNA gene & mRNA splicing, SUMOylation \\
\hline NAA35 & $\begin{array}{l}\text { N(Alpha)-Acetyltransferase 35, } \\
\text { NatC Auxiliary Subunit }\end{array}$ & rs1114081, rs2277193 & $\begin{array}{l}\text { Involved in the regulation of apoptosis, and the } \\
\text { proliferation of smooth muscle cells }\end{array}$ & Protein coding & $\begin{array}{l}\text { Golgi-to-endoplasmic reticulum (ER), } \\
\text { trans-Golgi-network retrograde transport }\end{array}$ \\
\hline
\end{tabular}


In summary, the four NTRK2 SNPs that contributed most to the SNP component were situated near alternative stop codons (rs4877289 between exons 16 and 17, and the others between exons 19 and 20); these SNPs act on NTRK2, its neighboring SLC28A3 and AGTPBP1 genes, and other genes (HNRNPK, MIR7-1, NAA35, RM1) in and outside of the brain tissue. These genes are implicated in apoptosis regulation, smooth muscle cell proliferation, mRNA splicing, neurotransmission, translational control, SUMOylation, Golgi and ER retrograde transport, and the shortening of long polyglutamate chains, among other functions (Table 4). The effects of these NTRK2 SNPs on genes are most common and pronounced in the frontal cortex (in keeping with the SNP component's connection to frontal gray matter), followed by the thalamus, putamen, and cerebellum. Table 5 summarizes histone modifications and altered regulatory motifs associated with these SNPs.

Table 5. Histone modifications contributing to chromatin states in the top NTRK2 SNPs in the brain. Altered regulatory motifs associated with SNPs are also listed. H3K27ac = enhancer/ promoter-associated, H3K4me1 = enhancer-associated. CG = cingulate gyrus, IT = inferior temporal, $\mathrm{AG}=$ angular gyrus, DLPFC $=$ dorsolateral prefrontal cortex, Ant. Caud. = anterior caudate, MHipp = middle hippocampus, SNigra = substantia nigra. Data from HaploReg v4.1 [41].

\begin{tabular}{|c|c|c|c|c|c|c|c|c|}
\hline $\begin{array}{l}\text { NTRK2 } \\
\text { SNP }\end{array}$ & CG & IT & AG & DLPFC & $\begin{array}{l}\text { Ant. } \\
\text { Caud. }\end{array}$ & MHipp & SNigra & $\begin{array}{c}\text { Regulatory } \\
\text { Motifs Altered }\end{array}$ \\
\hline rs11140810 & H3К27ac & H3К27ac & H3К27ac & Н3К27ас & & & & $\begin{array}{c}\text { Foxa, GLI, Hic1, } \\
\text { Zec }\end{array}$ \\
\hline rs4877289 & H3K4me1 & & & & H3K4me1 & & & \\
\hline rs10868241 & $\begin{array}{l}\text { H3K27ac, } \\
\text { H3K4me1 }\end{array}$ & $\begin{array}{l}\text { H3K27ac, } \\
\text { H3K4me1 }\end{array}$ & H3К27ac & $\begin{array}{l}\text { H3K27ac, } \\
\text { H3K4me1 }\end{array}$ & Н3К27ac & $\begin{array}{l}\text { H3K27ac, } \\
\text { H3K4me1 }\end{array}$ & $\begin{array}{l}\text { H3K27ac, } \\
\text { H3K4me1 }\end{array}$ & \\
\hline rs2277193 & Н3К27ac & Н3К27ac & Н3К27ac & Н3К27ac & & & & GR, Pax-6 \\
\hline
\end{tabular}

It is important to consider LD pruning when interpreting these results. Although two of these SNPs (rs1114081 and rs2277193) are not in high LD with other SNPs, rs4877289 is in high LD with three SNPs (rs10780691, rs10868235, rs10868230; $\left.r^{2}: 0.92-0.98\right)$ and rs10868241 is in high LD with nine SNPs (rs7858707, rs10116596, rs10122796, rs7030319, rs4329345, rs11464614, rs10780693, rs11140813, rs4457413; $\left.r^{2}: 0.97-1.0\right)$. Thus, the effects of the latter two SNPs in this study may be attributable to an SNP in high LD that was pruned from the dataset.

\subsection{Top Contributing SNPs Outside of NTRK2}

Although NTRK2 SNPs were disproportionally represented in the top component SNPs, other top SNPs included intronic CDK14, FAM114A1, and HEATR4 SNPs, as well as intergenic SNPs (rs548321 closest to LRRC55, rs427790 between MIR181A1 and NR5A2). Many of these genes are implicated in cancer regulation (Supplementary Materials Table S2). Overall, compared to top component NTRK2 SNPs, top SNPs outside of NTRK2 were less commonly associated with histone modifications in the brain, but were more often reported to influence regulatory motifs and gene expression outside the brain. In Braineac data, the 10 strongest SNP influences on brain gene expression among our top 10 component SNPs were exhibited by three of the SNPs outside of NTRK2 (rs7655305, rs8012614, rs548321); rs7655305 accounted for half of these effects. Supplementary Materials Table S3, a complement to Table 5, summarizes brain histone modifications and altered regulatory motifs associated with these SNPs (the HEATR4 SNP is omitted due to the absence of relevant findings). Of these SNPs, the intergenic SNPs rs548321 (LRRC55-associated) and rs427790 (MIR181A1 and NR5A2-associated) share perhaps the most overlap with the NTRK2 SNPs; rs2277193 (NTRK2) and rs548321 both alter GR (glucocorticoid receptor) and Pax (paired box) regulatory motifs (Pax-4 for rs548321 and Pax-6 for rs2277193) and are associated with histone changes in the brain. rs11140810 (NTRK2) and rs427790 alter the same Zec transcription factor and two distinct Fox regulatory motifs (Foxj1 for rs427790 and Foxa for rs11140810). Each of these SNPs are outlined in more detail below. 
The CDK14 variant (rs7801922) contributed to the SNP component more than any other SNP. This intronic SNP is 68,161 bp upstream of CDK14 exon 1, and is an eQTL in the lungs (GTPBP10), tibial artery (CDK14), tibial nerve (GTPBP10), and thyroid (DPY19L2P4) [43]. In the brain, rs7801922 is an eQTL for the DLPFC in psychiatric populations [39]. In healthy populations, this variant most affects genes that exhibit reduced expression in white matter relative to other brain tissue types (e.g., STEAP2, DPY19L2P4, C7orf63) [40]. Supplementary Materials Table S4 lists examples of gene regulation related to this SNP. rs7801922 is associated with altered regulatory motifs (ecotropic virus integration site (Evi-1), GATA-binding factor (GATA), interferon regulatory factor (Irf), and suppressor of essential function (SEF-1)), and is in LD with three other CDK14 SNPs associated with other regulatory motif alterations [41].

An intronic HEATR4 (HEAT-repeat containing 4) variant was another top SNP. HEAT-repeats are amino acid domains that often have roles in protein-protein interactions, and are considered important for intracellular transport, microtubule dynamics, and chromosomal separation. Huntingtin is one of the first proteins in which HEAT-repeats were discovered (HEAT stands for Huntingtin, Elongation factor 3, protein phosphatase 2A, and TOR) [44]. The HEATR4 SNP that was prominent in our genetic component (rs8012614) is an eQTL for ACOT4 (exon, expressed region, gene, and transcript levels), DNAL1 (expressed region level), and HEATR4 (expressed region level) in the DLPFC [39]; in controls, the SNP was an eQTL for ACOT4 only (exon, expressed region, and gene levels). Additionally, this SNP has more Genotype-Tissue Expression (GTEx) eQTL hits than any other top contributing SNP, although these are mostly outside the brain in adipose, muscle, skin, heart, pancreas, and thyroid tissue [43]. The effect of this SNP on DNAL1 is particularly interesting, as this gene is not only part of the Kyoto Encyclopedia of Genes and Genomes (KEGG) Huntington's disease gene pathway but is also directly involved in aberrant BDNF transport [45]. DNAL1 is one of three genes associated with dynein axonemal light chain 4, part of the ATP-dependent outer dynein arms complex that acts as a molecular motor for cilia [31].

The final intronic SNP, rs7655305, regulates the expression of FAM114A1 (mostly in the medulla, substantia nigra, and hippocampus) [40] and other genes, especially in the cerebellum (Table 3). This variant is an eQTL for TLR1 in the DLPFC (transcript and exon levels in the disease population) [39] as well as other genes in many areas outside the brain, including skin (especially sun-exposed), tibial nerve, subcutaneous and visceral adipose, breast mammary tissue, testis, thyroid, and esophagus. rs7655305 is associated with enhancer histone marks in four tissues outside of the brain, the alteration of CCCTC-binding factor (CTCF) and telomere length regulation (Tel2) regulatory motifs [41], and active H3K36me3 (transcription-associated) histone modification in the middle hippocampus (http:/ / www.featSNP.org). This SNP is also in LD with several other intronic FAM114A1 SNPs with correlated enhancer histone marks, altered regulatory motifs, and eQTL hits [41].

rs112140519 is a deletion/insertion variation closest to NUS1 that is linked to H3K4me3 promotion in adipose nuclei and duodenum smooth muscle as well as the alteration of five regulatory motifs (Dbx1, Hoxb13, Ncx, ZNF263, Zfp105) [41]. rs548321, an intergenic SNP 70 kbps from the $5^{\prime}$ end of LRRC55, most strongly affects the expression of TIMM10, UBE2L6, and ZDHHC5 (especially in the putamen, although TIMM10 effects were most prominent in the frontal cortex). Each of these genes exhibits the lowest expression in the cerebellum relative to other brain tissue types [40]. Haploreg data shows selective association with H3K4me1 and H3K27ac enhancements in anterior caudate, as well as the alteration of GR and Pax-4 regulatory motifs [41]. rs427790, an intergenic SNP between MIR181A1 and NR5A2, is an eQTL hit in the testis and basal ganglia [43]. Haploreg indicates the associated alteration of double homeodomain (Dux1), Foxj1, and Zec regulatory motifs [41].

\subsection{Influence of HTT CAG-Repeats}

Including the CAG-repeat number or CAG-age-product (CAP) score as a covariate did not affect the significance of the GMC-SNP component correlation. Similarly, the inclusion of the CAP did not nullify the significance of the NTRK2-SNP correlation with TMTB, although it did account for 
some of the variance. While we can currently only speculate on this finding, it may be attributable to the regression of age effects from the imaging data prior to PICA. The CAP reflects the cumulative disease burden of age and the CAG-repeat number. Because the GMC component had the effects of age subtracted from it, CAP and CAG would be expected to display a similar relationship to the GMC component, which is what we observed. The relationships between the GMC component and the clinical variables, however, could be accounted for by the CAP, likely reflecting the sensitivity of these measures to CAG and age, both of which are highly related to the clinical variables assessed in this study.

\section{Conclusions}

The study results demonstrated the following:

1. In this PREDICT-HD prodromal cohort, high or low levels of an SNP profile with substantial contributions from NTRK2 were associated with a GMC profile representing the supplementary and primary motor cortex, as well as other frontal regions (positive correlation).

2. This frontal gray matter profile was associated with cognitive and motor performance in this population.

3. The SNP component was not significantly associated with clinical functioning, but one of its top NTRK2 SNPs had a protective association with performance on TMTB, a measure of task switching and visual attention, indicating some influence on cognition.

4. Correlations between the SNP component and clinical/GMC variables were mainly due to top contributing SNPs, rather than being an aggregate effect of the entire SNP component.

5. Top component SNPs have been associated with active histone modifications in the brain (cingulate gyrus, inferior temporal, angular gyrus, DLPFC, caudate, hippocampus, and substantia nigra) and altered regulatory motifs (especially the glucocorticoid receptor (GR) and zinc finger protein (Zec)).

6. Top NTRK2 SNPs in the component were close to alternative stop codons and reportedly regulated genes implicated in diverse functions (especially in the frontal cortex, thalamus, putamen, and cerebellum).

Although further investigation is warranted, these results suggest that NTRK2 has protective potential in Huntington's disease, especially in individuals with certain genotypes. Treatments that target BDNF receptors may help preserve frontal gray matter that is important for cognitive and motor functioning.

\section{Limitations}

In any study involving many participants with a rare condition, multiple scanning sites are typical and a certain degree of inhomogeneity in data collection is thus inevitable. This study incorporates data from several unique $1.5 \mathrm{~T}$ and $3 \mathrm{~T}$ MRI scanners, and every effort was made to control for possible confounds related to this. Before data collection, uniform protocols were established to ensure maximal homogeneity of data collection. The effects of collection site and scanner field strength, as well as sex and age, were regressed from GMC images before pICA.

Ideally, controls would be included in the analysis to permit comparisons with prodromal HD participants. PICA was carried out with a more extensive dataset $(N=903)$ that included controls $(N=189)$. However, the analysis did not extract a significantly correlated SNP-GMC component pair that withstood correction for multiple testing. The effects presented in this study may be unapparent in the combined sample with controls because they are related to other processes already aberrant in $\mathrm{HD}$, such as BDNF transcription and nuclear translocation.

Because the GMC-SNP component correlation was driven by a small percentage of participants, the results may not apply to the entire HD population, and treatments that target NTRK2 should consider the context of the patient's genomic landscape. Nonetheless, for cancer and other diseases, 
emphasis is shifting away from blanket treatments toward more personalized gene therapies that consider individual genotypes. If clinical trials to reduce $\mathrm{mHTT}$ are ineffective or harmful for certain individuals in the long-run, therapies that instead target mediators of mHTT toxicity may provide a promising alternative.

\section{Future Directions}

This study helped characterize the complex interactions of BDNF-signaling genes, brain structure, and clinical functioning in prodromal HD. As genetic and epigenetic factors of prodromal HD progression continue to be identified, potential gene therapies (such as DNA-methylating drugs and histone-deacetylase inhibitors) can be refined, tested, and implemented more strategically [46]. To aid the development of targeted treatments, future studies should test the validity of strong correlations observed in the most extensive available human datasets by establishing causal links in non-human animals. For example, many HD Drosophila (fruit fly) strains are available that permit rapid, efficient, and inexpensive querying of SNPs and genes of interest derived from human studies. The most promising candidates from these experiments can be flagged for continued research in mammals and eventual use in clinical trials. A promising direction is to examine how different ratios of BDNF TrkB and p75 receptors influence progression and onset in the context of HTT CAG-repeats. BDNF acts on TrkB receptors to increase its own continued expression; thus, an underrepresentation of these receptors may reduce BDNF. Similarly, p75 receptor overrepresentation or an overabundance of pro-BDNF may promote apoptosis and contribute to disease-related decline. Another important consideration is the impact of other BDNF exons. Huntingtin stimulates transcription from the BDNF exon II promoter, which is $60 \%$ less active in cells overexpressing mHTT [9]. Although BDNF exon III and IV promoter actions are not linked to huntingtin, $\mathrm{mHTT}$ reduces their transcription through unknown mechanisms thought to involve cAMP Response Element Binding protein (CREB) and CREB-Binding Protein (CBP). Thus, promoter II transcriptional reduction may be linked to huntingtin reductions, while that of promoters III and IV may be related to mHTT toxicity. CREB and CBP are also linked to several altered functions in HD, and their investigation is a valuable avenue for future research. This study examined BDNF-signaling genes in relation to GMC and clinical functioning. However, white matter is dramatically affected in HD and may also be influenced by these factors, a possibility that should be examined in future studies. Aside from genetic influences on HD progression, epigenetic marks (which can alter gene expression) also differ in patients compared to controls. This may be an especially promising therapeutic direction because, unlike the fixed DNA sequence, the epigenome changes across the lifespan and in response to environmental stimuli. Future studies should seek to characterize prodromal epigenetic differences in various tissues, as well as identify protective and detrimental variations in pathways with known clinical relevance to HD. An additional important future direction is to characterize these factors longitudinally. SNP and methylation patterns, for example, in BDNF/REST pathways may be associated with different rates of clinical decline or the rapidity of HD onset. Factors associated with disease resilience could then be incorporated into intervention strategies to slow or prevent HD conversion.

Supplementary Materials: The following are available online at http:/ /www.mdpi.com/2076-3425/8/7/116/s1, Table S1: Top 61 SNPs contributing to the frontal GMC component, Figure S1: Regression influence analysis in R to identify participants driving the significant frontal GMC - NTRK2 SNP profile correlation, Table S2: Descriptions of genes affected by top contributing component SNPs outside of NTRK2, Table S3: Associated active histone modifications contributing to chromatin states in the brain at top component SNPs outside of NTRK2, Table S4: Reported gene regulation by rs7801922, the top SNP contributing to the frontally-related SNP component, in and outside of brain tissue.

Author Contributions: Conceptualization, J.C., J.L., and J.T.; data curation, J.L., H.J., H.B., and S.P.; formal analysis, J.C.; funding acquisition, V.C., J.T., and J.P.; investigation, J.C.; methodology, J.C., J.L., and V.C.; project administration, H.J., H.B., S.P., and J.P.; resources, J.L., V.C., J.T., and J.P.; software, J.C., J.L., and V.C.; supervision, J.L., J.T., and J.P.; validation, J.C.; visualization, J.C.; writing-original draft, J.C.; writing-review and editing, J.C., J.L., V.C., H.J., M.M., F.E., A.C., J.T., and J.P. PREDICT-HD Investigators, Coordinators, Motor Raters, Cognitive Raters, Executive Committee, Principal Investigator Jane S. Paulsen, Jeffrey D. Long, Hans J. Johnson, Thomas 
Brashers-Krug, Phil Danzer, Amanda Miller, H. Jeremy Bockholt, and Kelsey Montross. Scientific Consultants, Deborah Harrington (University of California, San Diego); Holly Westervelt (Rhode Island Hospital/Alpert Medical School of Brown University); Elizabeth Aylward (Seattle Children's Research Institute); Stephen Rao (Cleveland Clinic); David J. Moser, Janet Williams, Nancy Downing, Vincent A. Magnotta, Hans J. Johnson, Thomas Brashers-Krug, Jatin Vaidya, Daniel O'Leary, and Eun Young Kim (University of Iowa). Core Sections, Biostatistics: Jeffrey D. Long, Ji-In Kim, Spencer Lourens (University of Iowa); Ying Zhang and Wenjing Lu (University of Indiana). Ethics: Cheryl Erwin (Texas Tech University Health Sciences Center); Thomas Brashers-Krug, Janet Williams (University of Iowa); and Martha Nance (University of Minnesota). Biomedical Informatics: H. Jeremy Bockholt, Jason Evans, and Roland Zschiegner (University of Iowa). PREDICT-HD Coinvestigators, Amanda Miller, LMSW (University of Iowa, Iowa City, Iowa, USA, site investigator); Edmond Chiu, MD, and Samantha Loi, FRANZCP, MBBS, BMedSc (St. Vincent's Hospital, The University of Melbourne, Kew, Victoria, Australia, site investigators); Phyllis Chua, MD (The University of Melbourne, Royal Melbourne Hospital, Melbourne, Australia, site investigator); Lynn Raymond, MD, PhD (University of British Columbia, Vancouver, British Columbia, Canada, site investigator); Christopher A. Ross, MD, PhD (Johns Hopkins University, Baltimore, Maryland, USA, site investigator); William M. Mallonee, MD (Hereditary Neurological Disease Centre, Wichita, Kansas, USA, site investigator); Ali Samii, MD (University of Washington and VA Puget Sound Health Care System, Seattle, Washington, USA, site investigator); Randi Jones, PhD (Emory University School of Medicine, Atlanta, Georgia, USA, site investigator); Roger A. Barker, BA, MBBS, MRCP (John van Geest Centre for Brain Repair, Cambridge, UK, site investigator); Elizabeth McCusker, MD, and Clement Loy, MD (Westmead Hospital, Sydney, Australia, site investigators); Michael Orth, MD, PhD, and Sigurd Süßmuth, MD (University of Ulm, Ulm, Germany, site investigators); Kimberly Quaid, PhD (Indiana University School of Medicine, Indianapolis, IN, USA, site investigator); Mark Guttman, MD (Centre for Addiction and Mental Health, University of Toronto, Markham, Ontario, Canada, site investigator); Susan Perlman, MD (UCLA Medical Center, Los Angeles, California, USA, site investigator); Michael D. Geschwind, MD, PhD and Sharon Sha, MD (University of California, San Francisco, California, USA, site investigators); Tom Warner, MD, PhD (National Hospital for Neurology and Neurosurgery, London, UK, site investigator); Anne Rosser, MD, PhD, MRCP (Cardiff University, Cardiff, Wales, UK, site investigator); Frederick Marshall, MD (University of Rochester, Rochester, New York, USA, site investigator); Peter Panegyres, MB, BS, PhD, and Joseph Lee, MBBS, DPM, MCIL, MRCPsych, FHKCPsych, FHKAM (Psych), FRANZCP (Neurosciences Unit, Graylands, Selby-Lemnos \& Special Care Health Services, Perth, Australia, site investigators); Joel Perlmutter, MD (Washington University, St. Louis, Missouri, USA, site investigator); Zosia Miedzybrodzka, MD, PhD, (Clinical Genetics Centre, Aberdeen, Scotland, UK, site investigator); David Craufurd, MD (University of Manchester, Manchester, UK, site investigator); Pietro Mazzoni, MD, PhD (site investigator), and Karen Marder, MD, PhD (administrative investigator) (Columbia University Medical Center, New York, New York, USA); Rajeev Kumar, MD, (Colorado Neurological Institute, Englewood, Colorado, USA, site investigator); Vicki Wheelock, MD (University of California, Davis, Sacramento, California, USA, site investigator); Wayne Martin, MD, and Oksana Suchowersky, MD (University of Alberta, Edmonton, Alberta, Canada, site investigators); Anwar Ahmed, MD (site investigator), and Stephen Rao, MD (administrative investigator) (Cleveland Clinic Foundation, Cleveland, Ohio, USA). PREDICT-HD Contributors, Isabella De Soriano and Courtney Shadrick (University of Iowa, Iowa City, Iowa, USA, site coordinators); Joy Preston (site coordinator), Anita Goh (site coordinator), and Stephanie Antonopoulos (cognitive rater) (St. Vincent's Hospital, The University of Melbourne, Kew, Victoria, Australia); Angela Komiti (The University of Melbourne, Royal Melbourne Hospital, Melbourne, Australia, site coordinator); Joji Decolongon (site coordinator), Mannie Fan (site coordinator), and Allison Coleman (cognitive rater) (University of British Columbia, Vancouver, British Columbia, Canada); Mark Varvaris (cognitive rater), Maryjane Ong (site coordinator), and Nadine Yoritomo (site coordinator) (Johns Hopkins University, Baltimore, Maryland, USA); Greg Suter (Hereditary Neurological Disease Centre, Wichita, Kansas, USA, site coordinator); Emily P. Freney and Alma Macaraeg (University of Washington and VA Puget Sound Health Care System, Seattle, Washington, USA, site coordinators); Cathy Wood-Siverio (site coordinator) and Stewart A. Factor (motor rater) (Emory University School of Medicine, Atlanta, Georgia, USA); Sarah Mason (site coordinator) and Natalie Valle Guzman (cognitive rater) (John van Geest Centre for Brain Repair, Cambridge, UK); Jane Griffith, Jillian McMillan, and David Gunn (Westmead Hospital, Sydney, Australia, site coordinators); Katrin Barth (site coordinator), Sonja Trautmann (site coordinator), Daniela Schwenk (cognitive rater), and Carolin Eschenbach (cognitive rater) (University of Ulm, Ulm, Germany); Melissa Wesson (site coordinator) and Joanne Wojcieszek (motor rater) (Indiana University School of Medicine, Indianapolis, IN, USA); Alanna Sheinberg (cognitive rater), Albie Law (site coordinator), and Irita Karmalkar (site coordinator) (Centre for Addiction and Mental Health, University of Toronto, Markham, Ontario, Canada); Brian Clemente (UCLA Medical Center, Los Angeles, California, USA, site coordinator); Joseph Winer and Gabriela Satris (University of California, San Francisco, California, USA, site coordinators); Maggie Burrows (National Hospital for Neurology and Neurosurgery, London, UK, site coordinator); Kathy Price and Sarah Hunt (Cardiff University, Cardiff, Wales, UK, site coordinators); Amy Chesire (site coordinator), Mary Wodarski (cognitive rater), and Charlyne Hickey (motor rater) (University of Rochester, Rochester, New York, USA); Maria Tedesco and Brenton Maxwell (Neurosciences Unit, Graylands, Selby-Lemnos \& Special Care Health Services, Perth, Australia, site coordinators); Stacey Barton and Shineeka Smith (Washington University, St. Louis, Missouri, USA, site coordinators); Daniela Rae, Vivien Vaughan, and Mariella D'Alessandro (Clinical Genetics Centre, Aberdeen, Scotland, UK, site investigators); Judith Bek (site coordinator) and Elizabeth Howard (motor rater) (University of Manchester, Manchester, UK); Paula Wasserman (Columbia University Medical Center, New York, New York, USA, site coordinator); Diane Erickson (site coordinator), Christina Reeves (site coordinator), and Breanna Nickels (cognitive rater) (Colorado Neurological Institute, Englewood, Colorado, USA); Lisa Kjer (site coordinator), Amanda Martin (site coordinator), and Sarah Farias (cognitive rater) (University 
of California, Davis, Sacramento, California, USA); Pamela King (site coordinator), Marguerite Wieler (site coordinator), and Satwinder Sran (cognitive rater) (University of Alberta, Edmonton, Alberta, Canada); Christine Reece, Alex Bura, and Lyla Mourany (Cleveland Clinic Foundation, Cleveland, Ohio, USA, site coordinators).

Funding: This project was supported by 1U01NS082074 (V.C. and J.T., co-principal investigators) from the National Institutes of Health, National Institute of Neurological Disorders and Stroke. The PREDICT-HD study was supported by NIH/NINDS grant 5R01NS040068 awarded to J.P.; CHDI Foundation, Inc., A3917 and 6266 awarded to J.P.; Cognitive and Functional Brain Changes in Preclinical Huntington's Disease (HD) 5R01NS054893 awarded to J.P.; 4D Shape Analysis for Modeling Spatiotemporal Change Trajectories in Huntington's 1U01NS082086; Functional Connectivity in Premanifest Huntington's Disease 1U01NS082083; and Basal Ganglia Shape Analysis and Circuitry in Huntington's Disease 1U01NS082085 awarded to Christopher A. Ross.

Conflicts of Interest: The authors declare no conflict of interest. The founding sponsors had no role in the design of the study; in the collection, analyses, or interpretation of data; in the writing of the manuscript, and in the decision to publish the results.

\section{References}

1. Paulsen, J.S.; Long, J.D.; Johnson, H.J.; Aylward, E.H.; Ross, C.A.; Williams, J.K.; Nance, M.A.; Erwin, C.J.; Westervelt, H.J.; Harrington, D.L. Clinical and biomarker changes in premanifest huntington disease show trial feasibility: A decade of the predict-hd study. Front. Aging Neurosci. 2014, 6, 78. [CrossRef] [PubMed]

2. Rincon-Limas, D.E.; Jensen, K.; Fernandez-Funez, P. Drosophila models of proteinopathies: The little fly that could. Curr. Pharm. Des. 2012, 18, 1108-1122. [PubMed]

3. Long, J.D.; Paulsen, J.S. PREDICT-HD Investigators and Coordinators of the Huntington Study Group. Multivariate prediction of motor diagnosis in huntington's disease: 12 years of predict-hd. Mov. Disord. 2015, 30, 1664-1672. [CrossRef] [PubMed]

4. Binder, D.K.; Scharfman, H.E. Brain-derived neurotrophic factor. Growth Factors 2004, 22, 123-131. [CrossRef] [PubMed]

5. Hamada, K.; Terauchi, A.; Nakamura, K.; Higo, T.; Nukina, N.; Matsumoto, N.; Hisatsune, C.; Nakamura, T.; Mikoshiba, K. Aberrant calcium signaling by transglutaminase-mediated posttranslational modification of inositol 1,4,5-trisphosphate receptors. Proc. Natl. Acad. Sci. USA 2014, 111, E3966-E3975. [CrossRef] [PubMed]

6. Fan, J.; Cowan, C.M.; Zhang, L.Y.; Hayden, M.R.; Raymond, L.A. Interaction of postsynaptic density protein-95 with nmda receptors influences excitotoxicity in the yeast artificial chromosome mouse model of huntington's disease. J. Neurosci. 2009, 29, 10928-10938. [CrossRef] [PubMed]

7. Buckley, N.J.; Johnson, R.; Zuccato, C.; Bithell, A.; Cattaneo, E. The role of rest in transcriptional and epigenetic dysregulation in huntington's disease. Neurobiol. Dis. 2010, 39, 28-39. [CrossRef] [PubMed]

8. Reddy, P.H.; Mao, P.; Manczak, M. Mitochondrial structural and functional dynamics in huntington's disease. Brain Res. Rev. 2009, 61, 33-48. [CrossRef] [PubMed]

9. Zuccato, C.; Cattaneo, E. Role of brain-derived neurotrophic factor in huntington's disease. Prog. Neurobiol. 2007, 81, 294-330. [CrossRef] [PubMed]

10. Pearlson, G.D.; Liu, J.; Calhoun, V.D. An introductory review of parallel independent component analysis ( $\mathrm{p}$-ica) and a guide to applying p-ica to genetic data and imaging phenotypes to identify disease-associated biological pathways and systems in common complex disorders. Front. Genet. 2015, 6, 276. [CrossRef] [PubMed]

11. Chen, J.; Calhoun, V.D.; Ulloa, A.E.; Liu, J. Parallel ica with multiple references: A semi-blind multivariate approach. In Proceedings of the 2014 36th Annual International Conference of the IEEE Engineering in Medicine and Biology Society, Chicago, IL, USA, 26-30 August 2014; pp. 6659-6662.

12. Gupta, C.N.; Chen, J.; Liu, J.; Damaraju, E.; Wright, C.; Perrone-Bizzozero, N.I.; Pearlson, G.; Luo, L.; Michael, A.M.; Turner, J.A. Genetic markers of white matter integrity in schizophrenia revealed by parallel ica. Front. Hum. Neurosci. 2015, 9, 100. [CrossRef] [PubMed]

13. Laforce, R.; Tosun, D.; Ghosh, P.; Lehmann, M.; Madison, C.M.; Weiner, M.W.; Miller, B.L.; Jagust, W.J.; Rabinovici, G.D. Parallel ica of fdg-pet and pib-pet in three conditions with underlying alzheimer's pathology. Neuroimage Clin. 2014, 4, 508-516. [CrossRef] [PubMed]

14. BDNF in Huntington's Disease: Role in Pathogenesis and Treatment. Available online: http://cdn. intechopen.com/pdfswm/28345.pdf (accessed on 22 June 2018). 
15. Paulsen, J.S.; Langbehn, D.R.; Stout, J.C.; Aylward, E.; Ross, C.A.; Nance, M.; Guttman, M.; Johnson, S.; MacDonald, M.; Beglinger, L.J. Detection of huntington's disease decades before diagnosis: The predict-hd study. J. Neurol. Neurosurg. Psychiatry 2008, 79, 874-880. [CrossRef] [PubMed]

16. Paulsen, J.S.; Long, J.D.; Ross, C.A.; Harrington, D.L.; Erwin, C.J.; Williams, J.K.; Westervelt, H.J.; Johnson, H.J.; Aylward, E.H.; Zhang, Y. Prediction of manifest huntington's disease with clinical and imaging measures: A prospective observational study. Lancet Neurol. 2014, 13, 1193-1201. [CrossRef]

17. Wechsler, D. Manual for the Wechsler Adult Intelligence Scale—Revised; Psychological Corporation: New York, NY, USA, 1981.

18. Lezak, M.D.; Howieson, D.; Loring, D. Neuropsychological Assessment, 4th ed.; Oxford University Press: New York, NY, USA, 2004.

19. Smith, A. Symbol Digit Modalities Test (SDMT) Manual (Revised); Western Psychological Services: Los Angeles, CA, USA, 1982.

20. Stroop, J.R. Studies of interference in serial verbal reactions. J. Exp. Psychol. 1935, 18, 643-662. [CrossRef]

21. Golden, C. Stroop color and word test: Cat. No. 30150m. In A Manual for Clinical and Experimental Uses; Stoelting: Chicago, IL, USA, 1978.

22. Reitan, R. Validity of the trail making test as an indicator of organic brain damage. Percept. Mot. Skills 1958, 8, 271-276. [CrossRef]

23. O’Rourke, J.J.; Beglinger, L.J.; Smith, M.M.; Mills, J.; Moser, D.J.; Rowe, K.C.; Langbehn, D.R.; Duff, K.; Stout, J.C.; Harrington, D.L. The trail making test in prodromal huntington disease: Contributions of disease progression to test performance. J. Clin. Exp. Neuropsychol. 2011, 33, 567-579. [CrossRef] [PubMed]

24. Genetic Modifiers of Huntington's Disease (GeM-HD) Consortium. Identification of genetic factors that modify clinical onset of huntington's disease. Cell 2015, 162, 516-526.

25. Kim, E.Y.; Magnotta, V.A.; Liu, D.; Johnson, H.J. Stable atlas-based mapped prior (stamp) machine-learning segmentation for multicenter large-scale mri data. Magn. Reson. Imaging 2014, 32, 832-844. [CrossRef] [PubMed]

26. Chen, J.; Calhoun, V.D.; Arias-Vasquez, A.; Zwiers, M.P.; van Hulzen, K.; Fernández, G.; Fisher, S.E.; Franke, B.; Turner, J.A.; Liu, J. G-protein genomic association with normal variation in gray matter density. Hum. Brain Mapp. 2015, 36, 4272-4286. [CrossRef] [PubMed]

27. Corp, I. Ibm SPSS Statistics for Windows, 21.0; IBM Corp: Armonk, NY, USA, 2012.

28. Chen, J.; Calhoun, V.D.; Liu, J. Ica order selection based on consistency: Application to genotype data. In Proceedings of the 2012 Annual International Conference of the IEEE Engineering in Medicine and Biology Society, San Diego, CA, USA, 28 August-1 September 2012; pp. 360-363.

29. Fox, J. Applied Regression Analysis and Generalized Linear Models, 2nd ed.; Sage Publications: Thousand Oaks, CA, USA, 2008.

30. Fox, J.; Weisberg, S. An R Companion to Applied Regression, 2nd ed.; Sage Publications: Thousand Oaks, CA, USA, 2011.

31. Stelzer, G.; Rosen, N.; Plaschkes, I.; Zimmerman, S.; Twik, M.; Fishilevich, S.; Stein, T.I.; Nudel, R.; Lieder, I.; Mazor, Y. The genecards suite: From gene data mining to disease genome sequence analyses. Curr. Protoc. Bioinform. 2016, 54, 1.30.1-1.30.33.

32. Zakzanis, K.K.; Mraz, R.; Graham, S.J. An fmri study of the trail making test. Neuropsychologia 2005, 43, 1878-1886. [CrossRef] [PubMed]

33. Moll, J.; de Oliveira-Souza, R.; Moll, F.T.; Bramati, I.E.; Andreiuolo, P.A. The cerebral correlates of set-shifting: An fmri study of the trail making test. Arq. Neuropsiquiatr. 2002, 60, 900-905. [CrossRef] [PubMed]

34. Galer, S.; Op De Beeck, M.; Urbain, C.; Bourguignon, M.; Ligot, N.; Wens, V.; Marty, B.; Van Bogaert, P.; Peigneux, P.; De Tiège, $X$. Investigating the neural correlates of the stroop effect with magnetoencephalography. Brain Topogr. 2015, 28, 95-103. [CrossRef] [PubMed]

35. Leung, H.C.; Skudlarski, P.; Gatenby, J.C.; Peterson, B.S.; Gore, J.C. An event-related functional mri study of the stroop color word interference task. Cereb. Cortex 2000, 10, 552-560. [CrossRef] [PubMed]

36. Grazioli, E.; Yeh, A.; Benedict, R.; Parrish, J.; Weinstock-Guttman, B. Cognitive dysfunction in ms: Bridging the gap between neurocognitive deficits, neuropsychological batteries and mri. Future Neurol. 2008, 3, 49-59. [CrossRef]

37. Nachev, P.; Kennard, C.; Husain, M. Functional role of the supplementary and pre-supplementary motor areas. Nat. Rev. Neurosci. 2008, 9, 856-869. [CrossRef] [PubMed] 
38. Stoilov, P.; Castren, E.; Stamm, S. Analysis of the human trkb gene genomic organization reveals novel trkb isoforms, unusual gene length, and splicing mechanism. Biochem. Biophys. Res. Commun. 2002, 290, 1054-1065. [CrossRef] [PubMed]

39. BrainSeq: A Human Brain Genomics Consortium. Brainseq: Neurogenomics to drive novel target discovery for neuropsychiatric disorders. Neuron 2015, 88, 1078-1083.

40. Ramasamy, A.; Trabzuni, D.; Guelfi, S.; Varghese, V.; Smith, C.; Walker, R.; De, T.; Coin, L.; de Silva, R.; Cookson, M.R. Genetic variability in the regulation of gene expression in ten regions of the human brain. Nat. Neurosci. 2014, 17, 1418-1428. [CrossRef] [PubMed]

41. Ward, L.D.; Kellis, M. Haploreg: A resource for exploring chromatin states, conservation, and regulatory motif alterations within sets of genetically linked variants. Nucleic Acids Res. 2012, 40, D930-D934. [CrossRef] [PubMed]

42. The UniProt Consortium. Uniprot: The universal protein knowledgebase. Nucleic Acids Res. 2017, 45, D158-D169.

43. GTEx-Consortium. Human genomics. The genotype-tissue expression (gtex) pilot analysis: Multitissue gene regulation in humans. Science 2015, 348, 648-660.

44. Andrade, M.A.; Bork, P. Heat repeats in the huntington's disease protein. Nat. Genet. 1995, 11, $115-116$. [CrossRef] [PubMed]

45. Kanehisa, M.; Furumichi, M.; Tanabe, M.; Sato, Y.; Morishima, K. Kegg: New perspectives on genomes, pathways, diseases and drugs. Nucleic Acids Res. 2017, 45, D353-D361. [CrossRef] [PubMed]

46. Lee, J.; Hwang, Y.J.; Kim, K.Y.; Kowall, N.W.; Ryu, H. Epigenetic mechanisms of neurodegeneration in huntington's disease. Neurotherapeutics 2013, 10, 664-676. [CrossRef] [PubMed]

(C) 2018 by the authors. Licensee MDPI, Basel, Switzerland. This article is an open access article distributed under the terms and conditions of the Creative Commons Attribution (CC BY) license (http://creativecommons.org/licenses/by/4.0/). 